\title{
Implications of Accelerated Self-Healing as a Key Design Knob for Cross-Layer Resilience
}

\author{
Xinfei Guo*, Mircea R. Stan \\ Department of Electrical and Computer Engineering \\ University of Virginia \\ Charlottesville, VA 22904, USA \\ $\{$ xg2dt, mircea $\} @$ virginia.edu
}

\begin{abstract}
In this paper we propose a cross-layer accelerated self-healing (CLASH) system which "repairs" its wearout issues in a physical sense through accelerated and active recovery, by which wearout can be reversed while actively applying several accelerated self-healing techniques, such as high temperature and negative voltages. Different from previous solutions of coping with wearout issues (e.g. BTI) by "tolerating", "slowing down" or "compensating", which still leave the irreversible (permanent) wearout component unchecked, the proposed solution is able to fully avoid the irreversible wearout through periodic rejuvenation, and this is inspired by the explored frequency dependent behaviors of wearout and (accelerated and active) recovery based on measurements on FPGAs. We demonstrate a case where the chip can always be brought back to the fresh status by employing a pattern of 31-hour regular operation (under room temperature and nominal voltage) followed by a 1-hour accelerated selfhealing (under high temperature and negative voltage). The proposed system integrates the notions of accelerated self-healing across multiple layers of the system stack. At the circuit level, a negative voltage generator and heating elements are designed and implemented; at the architecture level, the core can be allocated in a way such that the dark silicon or redundant resources can be healed by active elements; at the system level, right balance of stress and accelerated/active recovery can be employed by the system scheduler to fully mitigate the wearout; various wearout sensors act as the media between different layers. Overall, these techniques work together to guarantee that the whole system performs for more of the time at higher levels of performance and power efficiency by fully taking advantage of the extra opportunities enabled by the accelerated self-healing.
\end{abstract}

Index Terms-Wearout, BTI, Accelerated self-healing, Frequency dependence, Cross-layer.

\section{INTRODUCTION}

The never-ending demand for higher performance and lower power consumption pushes the aggressive technology scaling and the appearance of emerging devices, while further downscaling leads to major challenges, among which wearout (or aging) has become a huge reliability threat. Bias Temperature Instability (BTI) has been accepted as one of the most dominant wearout factors causing lifetime reliability problems in the front-end of line (FEOL) by worsening metrics across the digital system hierarchy [1]-[4], with performance

*Corresponding author.

Address: High-performance Low-power (HPLP) Lab, Rice Hall 330, 85 Engineer's Way, Charlottesville, VA 22904, USA

Email address:xg2dt@virginia.edu (X. Guo),

mircea@virginia.edu (M. R. Stan)

Tel: +1-434-227-7800 degradation or intrinsic faults at the circuit level [5], errors at the architecture level [3] and failures at the system level [6]. Thus, dealing with wearout issues (such as BTI) needs to cross layers, where various techniques are necessary to be implemented - from device level up to the application level to work together to achieve the optimal lifetime and acceptable wearout levels with a low cost [2], [4], [7], [8].

In general, these cross-layer techniques can be divided into two categories. The first one is during the design phase, when the worst case wearout levels are estimated (e.g. based on application behaviors) and design margin is reserved by adding guardband at the circuit level (e.g. oversizing), it has been shown in [2], [9], [10] that the margin can be $>20 \%$ for a 10-year lifetime constraint, the added margins usually lead to large timing slacks and therefore wasteful power consumption especially during the initial lifetime. A second solution would be adaptive techniques during run time, where wearout induced variations are tracked and monitored by sensors, then various actuators are employed for compensating or adapting to the variations dynamically [11], [12], so the system is able to be designed for the average case. Such actuators are not unlike those for PVT variations, for example, they can be DVFS and/or body bias at the circuit level [13], cache sizing at the architecture level [11], task allocation at the system level or loop perforation at the application level [3], [11]. While due to that wearout is time dependent by nature, and will get worse and worse fundamentally as the system runs even though some reversible (recoverable) wearout might not fully accumulate until the end of lifetime, thus the adaptation might be able to guarantee that the system function correctly, but it either runs sluggishly or burns too much power. In addition, wearout sensors (the expected number for a future SoC can reach as many as hundreds [11]) need to be ON for tracking over the entire lifetime, and this will add unacceptable tracking power overhead.

A better solution would be to somehow "repair" wearout issues by reducing the actual variations. Since many dominant wearout mechanisms, such as BTI, are voltage dependent, so one way is to scale down the voltage stress, thus to alleviate wearout during the run time [14], but this solution will introduce huge performance overhead. An alternative way is to take advantage of the recovery property of BTI by generating more idle time for passive recovery (system unstressed when not in use) [15]. While passive recovery is usually slow 
and unpredictable, and it cannot be used to reduce margins effectively, thus it is sometimes even ignored when modeling wearout phenomena. Besides, even recoverable wearout, like BTI, usually has an irreversible part that can't be recovered within a reasonable time [16], [17]. Thus these irreversible components left by passive recovery will keep accumulating over the rest of the lifetime. Different from previous solutions, in this paper, we propose and demonstrate a set of accelerated self-healing techniques, by which recovery can be made active by reversing the directions of the stress (e.g. using positive Vgs instead of negative $V g s$ for PMOS) and can be accelerated (e.g. by increasing the temperature). Furthermore, by studying the recovery property of BTI extensively based on actual measurement on FPGAs, we explore the frequency dependent behaviors of wearout and (accelerated and active) recovery, and this leads to an optimal strategy that schedules the system proactively in a way that it is active only during the reversible phase of wearout until the irreversible wearout kicks in, and during sleep period, the reversible part of wearout can be healed within a short time through the accelerated self-healing techniques. Overall wearout issues are fully avoided, and the irreversible wearout becomes almost unobservable. This solution, with scheduled explicit accelerated self-healing periods ahead of any sign of stress, is simpler to implement across the system hierarchy with the novel circuit-architecture designs and system-level optimal scheduling. The system benefits from the cross-layer accelerated self-healing (CLASH) by operating for a longer time with higher performance in a refreshed mode, thus it will eventually lead to the significant reduction of the guardbands, and better cumulative metrics (e.g. average performance) as well. Since the notion of accelerated selfhealing is orthogonal to the existing adaptive solutions (e.g. [11]), so there are also good opportunities to combine them together to achieve the optimal cross-layer resilience in a more effective way with much lower cost.

The remaining of this paper is organized as follows. Section II discusses the background and the prior work on BTI wearout and recovery. The accelerated and active recovery techniques are presented in Section III. The frequency dependent behaviors of wearout and recovery are illustrated in Section IV. Section V presents the detailed analysis for the proposed solutions. A potential cross-layer accelerated selfhealing (CLASH) system that integrates the proposed solutions across the system stack is presented in Section VI, followed by the tradeoff analysis in Section VII. Section VIII concludes the paper.

\section{BACKGROUND AND RELATED WORK}

\section{A. BTI Wearout and Recovery Basics}

Among various wearout phenomena, BTI is one of the most dominant mechanisms [1], [18], which increases the absolute value of threshold voltage $(V t h)$ of transistors over time under stress (such as voltage stress), thus increasing the circuit delay and shortening circuit lifetime [5], [18]. Negative Bias Temperature Instability (NBTI) occurs under negative voltage stress (between gate and source) conditions and affects PMOS transistors. Similarly, Positive Bias Temperature Instability

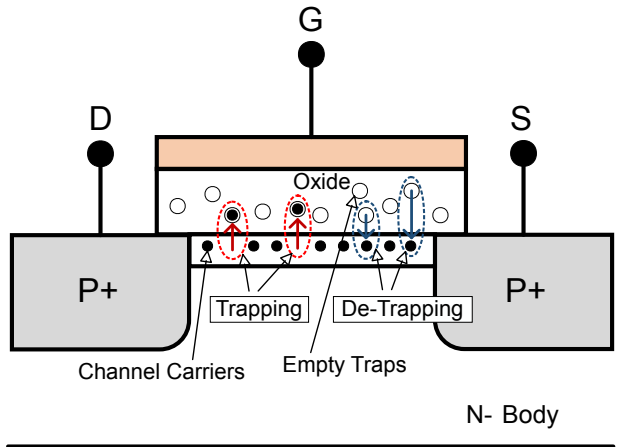

(a) BTI stress and recovery mechanisms

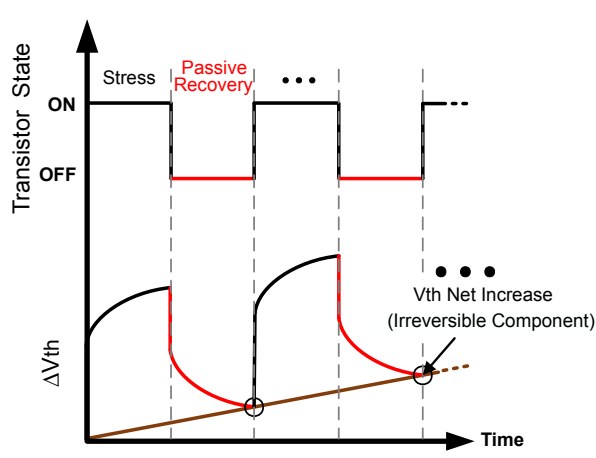

(b) Illustration of the device-level TD model

Fig. 1: BTI Recovery Basics.

(PBTI) affects NMOS transistors that are under positive stress voltage. Although the effect of PBTI has been negligible in previous technologies, it is rapidly becoming an important reliability issue with the introduction of high-k and metal gates [19]. Depending on the bias condition of the gate, there are two phases of BTI. As shown in Fig. 1b, the stress phase happens when the transistor is $\mathrm{ON}$, and the gate is under stress ( $\mathrm{Vgs}<0$ for PMOS, $\mathrm{Vgs}>0$ for NMOS), and the passive recovery phase starts when voltage stress is "released" $(\mathrm{Vgs}=0)$.

Although the mechanisms behind BTI is still somewhat controversial, yet the most likely explanation is that it is caused by trap generations at the $\mathrm{Si}-\mathrm{SiO}_{2}$ interface and in the oxide of the transistors [16], [18], [20]. The devicelevel trapping-detrapping (TD) model developed in [21], which has been validated against silicon and is widely used in the community, captures the details of the stress and passive recovery physically. According to the model, the threshold voltage of the transistor increases logarithmically when a trap captures a charge carrier in the stress phase (shown as Trapping process in Fig. 1a). If the transistor is in passive recovery phase, some of the interface traps can be annealed slowly (shown as De-Trapping process in Fig. 1a), and the number of occupied traps reaches a new equilibrium and results in partial recovery. Since the degradation effect of PBTI is similar to NBTI, the PBTI effect can be modeled similar to the NBTI effect [19]. Overall BTI effect on threshold voltage increase $\left(\Delta V_{t h}\right)$ follows equations from [21] and is shown in Fig. 1b. Assume that stress (denoted as $s t$ ) period for a fresh chip starts from time 0 , the threshold voltage increase until 
time $t_{s t}$ is modeled as:

$$
\Delta V_{t h}\left(t_{s t}\right)=\phi_{s t}\left(A+\log \left(1+C t_{s t}\right)\right)
$$

If a recovery (denoted as rec) interval of $t_{r e c}$ follows the stress phase, the total threshold voltage shift in the end is equal to:

$$
\begin{array}{r}
\Delta V_{t h}\left(t_{s t}+t_{r e c}\right)=\phi_{r e c}\left(A+\log \left(1+C t_{r e c}\right)\right)+ \\
\Delta V_{t h}\left(t_{s t}\right)\left(1-\frac{A+\log \left(1+C t_{r e c}\right)}{A+\log \left(1+C\left(t_{s t}+t_{r e c}\right)\right)}\right) \\
\phi_{s t / r e c} \sim K_{s t / r e c} \exp \left(\frac{-E}{k T_{s t / r e c}}\right) \exp \left(\frac{B V_{d d_{\text {st }} / r e c}}{k T_{\text {st/rec }} t_{\text {ox }}}\right)
\end{array}
$$

where $\phi_{s t}$ or $\phi_{\text {rec }}$ are functions of temperature and voltage under stress or recovery. A, B, C are (approximately) constant across the same technology node, $K_{s t}$ and $K_{r e c}$ are fitting parameters extracted from measurement conducted under different voltages/temperatures (will be discussed in Section III-B), k is Boltzmann's constant, $\mathrm{T}$ is temperature, $\mathrm{E}$ is activation energy, $t_{o x}$ is the oxide thickness, $V_{d d_{-} s t}$ and $V_{d d_{-} r e c}$ are supply voltages under stress and recovery, respectively. All parameters and their values used in this paper are summarized in Table I, and they are extracted based on our measurements which will be discussed in detail in the following sections. Equation (3) shows the strong dependence (exponentially) of the threshold voltage increase on voltage and temperature during both stress and recovery period.

TABLE I: Parameter descriptions for the model

\begin{tabular}{|c|c|c|}
\hline \hline Parameter & Decription & Value \\
\hline $\mathrm{k}$ & Boltzmann Constant & $1.38 \times 10^{-23} \mathrm{~J} / \mathrm{K}$ \\
$E$ & Activation Energy & $0.49 \mathrm{eV}$ \\
$t_{o x}$ & Oxide Thickness & $1 \mathrm{~nm}$ \\
$\mathrm{~A}$ & Constant & 0.2801 \\
$\mathrm{~B}$ & Constant & $3 \times 10^{-29}$ \\
$\mathrm{C}$ & Constant & 0.8614 \\
$K_{s t}$ & Fitting Parameter & $4.7 \times 10^{-4}$ \\
$K_{r e c}$ & Fitting Parameter & $7.34 \times 10^{-5}$ \\
\hline
\end{tabular}

\section{B. Prior Work on Wearout Recovery}

Although it has been shown in Fig. 1b that BTI can only be partially recovered under passive recovery condition, this property has been utilized in many work to improve the lifetime and other metrics (e.g. performance) of the system. Several methods [15], [22]-[24] were proposed to rebalance the signal probabilities to maximize the passive recovery time at the circuit level. An alternative method was to adaptively tune the performance according to the degree of wearout so that certain blocks could start the passive recovery phase earlier [25], [26]. Novel schemes were proposed to exploit the idle time of busy functional units for out-of-order processors [27] and superscalar [28]. A dynamic routing algorithm was proposed to adapt to the wearout-induced degradations in heterogeneous NoCs [29]. Since passive recovery is much slower than the wearout process [30], recovery boost for SRAM array was introduced in [31], [32], the idea was to raise the gate voltages of a memory cell in order to put PMOS devices into the recovery enhancement mode. A power napping scheme was proposed to help the recovery of NBTI and PBTI [33]. But

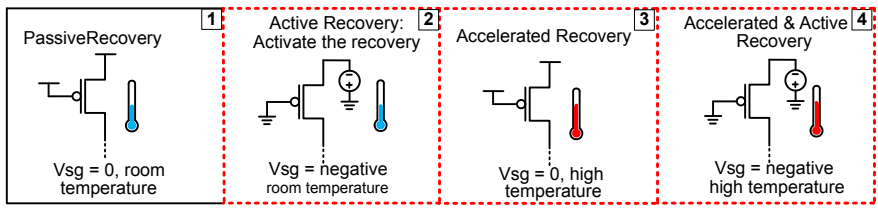

Fig. 2: Illustration of accelerated and active recovery techniques (NBTI case), No. 2, 3 and 4 are proposed accelerated self-healing solutions.

these work focus on only SRAM cell designs or architectural level implementations, it was still unclear how much benefit recovery boost could achieve due to lack of experimental data and models. In addition, these solutions still leave the irreversible wearout unchecked. Wafer level and transistor level experiments and theory [34]-[36], together with the device level model discussed in above section, indicated that BTI recovery highly depends on temperature, these work provided a physical evidence for our proposed accelerated self-healing techniques. Several recent work [17], [37] have studied the irreversible component of wearout at the device level. However, these work focused only on demonstrating and modeling the permanent component, thus a solution that could fundamentally repair the irreversible wearout is still missing in the field. The proposed solutions in this paper differ from the previous recovery and recovery boosting work in several aspects. Firstly, we demonstrate that both high temperature and negative voltage could accelerate recovery with actual measurements on 40nm FPGAs and also develop the analytic model for them. Secondly, we propose a biology-inspired strategy that runs the system in such a way that the irreversible wearout can be fully avoided so that the system keeps almost "fresh" all the time. Lastly, the proposed solutions can be effectively applied to all logic blocks, instead of just SRAM arrays, we show that the notion of accelerated self-healing can be implemented across the system hierarchy and be introduced as a key design knob for cross-layer resilience.

\section{Accelerated And ACtive Recovery}

In this paper, we propose that the systems could use their sleep time (e.g. intrinsic OFF periods) as an active recovery period essential for their overall performance, not unlike in biological world. During the sleep period, several accelerated self-healing ${ }^{1}$ solutions can be implemented to deeply rejuvenate the circuit, and they are shown in Fig. 2 (No. 2, 3 and 4). Different from the passive recovery (No. 1 in the figure), BTI recovery can be made active by "turning off" the transistor more via a negative voltage across the source and gate (No. 2). Secondly, high temperature can increase the kinetic energy for the charge carriers, thus leading to the accelerated recovery (No. 3). The third scenario is when active recovery can be further accelerated through the joint efforts of both negative voltage and high temperature (No. 4). We will present both analytic models and experimental demonstration of the proposed solutions in this section.

\footnotetext{
${ }^{1}$ Part of this work was originally published in [38].
} 
A. Gate-level Analytical Model for Accelerated and Active Recovery

To model the performance degradation and rejuvenation due to wearout and accelerated self-healing, a gate level analytical model that is based on the device model (1)-(3) is proposed in this section. Based on the circuit theory, the propagation delay of a digital gate can be approximated as:

$$
t_{d} \sim \frac{C_{L} V_{d d}}{I_{d}} \propto \frac{C_{L} V_{d d}}{V_{d d}-V_{t h}}
$$

where $C_{L}$ is the output capacitance of the gate. The change in gate delay when $V_{t h}$ is subject to change is:

$$
\Delta t_{d} \sim \frac{\Delta V_{t h}}{V_{d d}-V_{t h}} \cdot t_{d 0}
$$

where $t_{d 0}$ is the original delay of the gate without any $V_{t h}$ shift. Combine equations (1), (3) and (5), the total delay increase after $t_{s t}$ can be expressed as:

$\Delta t_{d}\left(t_{s t}\right) \sim \frac{\phi_{s t}}{V_{d d_{-} s t}} \exp \left(\frac{-E}{k T_{s t}}\right) \exp \left(\frac{B V_{d d_{-} s t}}{k T_{s t} t_{o x}}\right)\left(A+\log \left(1+C t_{s t}\right)\right)$

where $\phi_{s t}, A, B$ and $C$ have been discussed in Section II-A. During the accelerated self-healing phase, based on the recovery phase equation of the device model, we combine equations (2), (3) and (5), and the delay change after sleep period $t_{\text {rec }}$ becomes:

$$
\begin{array}{r}
\Delta t_{d}\left(t_{s t}+t_{r e c}\right) \sim \frac{\phi_{r e c}}{V_{d d_{-} r e c}}\left(A+\log \left(1+C t_{r e c}\right)\right)+ \\
\Delta t_{d}\left(t_{s t}\right)\left(1-\frac{A+\log \left(1+C t_{r e c}\right)}{A+\log \left(1+C\left(t_{s t}+t_{r e c}\right)\right)}\right)
\end{array}
$$

Assume that the ratio of operation time to active sleep time of the system is $\alpha$, the total time (stress time + sleep time) is $t_{\text {total }}$, based on (7), the overall delay increase will be:

$$
\begin{aligned}
& \Delta t_{d}\left(t_{\text {total }}\right) \sim \frac{\phi_{\text {rec }}}{V_{\text {dd_rec }}}\left(A+\log \left(1+C \frac{t_{\text {total }}}{1+\alpha}\right)\right)+ \\
& \Delta t_{d}\left(\frac{\alpha t_{\text {total }}}{1+\alpha}\right)\left(1-\frac{A+\log \left(1+C \frac{t_{\text {total }}}{1+\alpha}\right)}{A+\log \left(1+C t_{\text {total }}\right)}\right) \\
& \phi_{\text {rec }} \sim K_{\text {rec }} \exp \left(\frac{-E}{k T_{\text {rec }}}\right) \exp \left(\frac{B V_{\text {dd_rec }}}{k T_{\text {rec }} t_{\text {ox }}}\right)
\end{aligned}
$$

Several observations can be made based on the proposed model. Firstly, the exponential dependence of the total delay increase on recovery temperature and voltage shows that by increasing $T_{r e c}$ and decreasing $V_{d d_{-} r e c}$, the first component in Equation (8) can decrease significantly. The second observation is that the active vs. sleep ratio $\alpha$ also affects the overall delay change. The final observation is that the recovered part $\left(\Delta t_{d}\left(t_{\text {total }}\right)-\Delta t_{d}\left(t_{s t}\right)\right)$ highly depends on the previous stress history $\left(\Delta t_{d}\left(t_{s t}\right)\right)$.

\section{B. Test Setup}

1) Test Platform: FPGA vendors have been aggressive in adopting the very latest technology nodes; this makes FPGAs more susceptible to wearout that can lead to frequency degradation [39]. Due to their "bleeding edge" technologies, reconfigurability and regular structures, FPGAs are an ideal

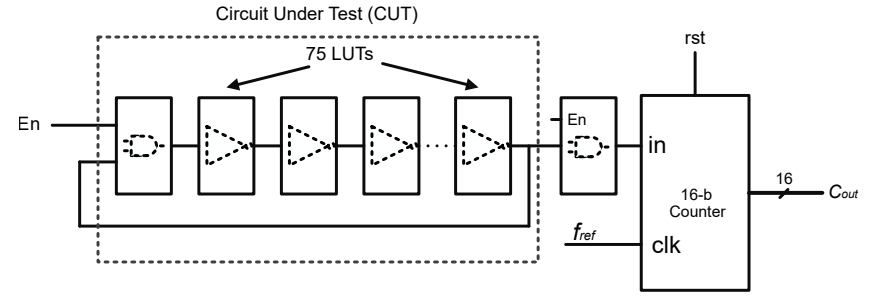

(a) Test Configuration

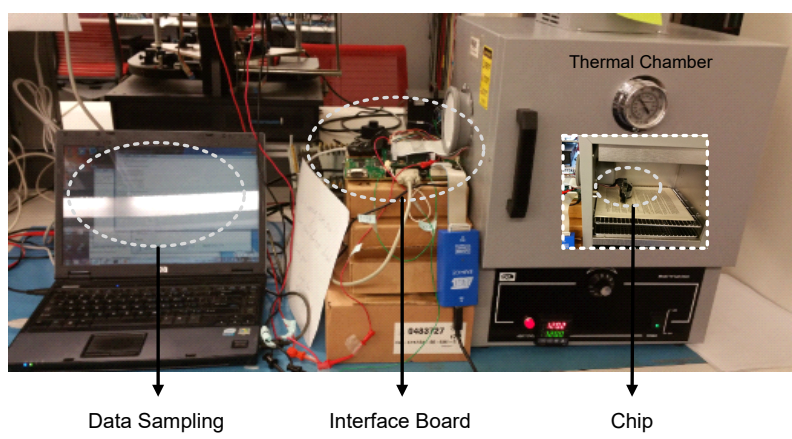

(b) Test Setup

Fig. 3: Experimental Setup.

test platform for wearout research [40]. In this paper, we use a type of 2-input Look Up Table (LUT)-based commercial FPGAs fabricated in a $40 \mathrm{~nm}$ technology to demonstrate the proposed accelerated and active recovery techniques.

2) Test Configuration: In the gate-level model, the delay change is used as the metric to capture the effect of wearout, thus the same metric is also used in the experimental part. We choose a ring oscillator (RO) structure which is widely used as a test platform [41] to measure the delay of the Circuit Under Test (CUT) to capture the delay change. Fig. 3a shows the test configuration, which is a LUT-based ring oscillator based on the design proposed in [42]. It consists of 75 inverters implemented in LUTs, and followed by a 16-bit counter to capture the output frequency of the ring oscillator. Enable signal En is used to switch between AC stress (switching) and DC (constant) stress mode. The oscillation frequency $f_{o s c}$ can be calculated as: $t_{d}=\frac{1}{2 f_{\text {osc }}}=\frac{1}{4 f_{\text {ref }} C_{\text {out }}}$, where $f_{\text {ref }}$ is the frequency of the reference clock. To pick this frequency, CUT is placed at different locations on the FPGA, and a diagnostic program is run. The output of the counter is read from a certain time range that has stable values. Environmental factors and the voltage supply are kept constant from one reading to another; when $f_{r e f}=500 \mathrm{~Hz}$, the variation of the counter output is within 5 and $0.0001 \%$ in terms of the corresponding $\mathrm{RO}$ frequency variation which we consider acceptable.

3) Test Conditions: Since both elevated temperature and/or voltage have a great impact on wearout and can be used to accelerate wearout. In this part of the work, only elevated temperature is applied since our preliminary tests show that we can observe a larger than $1 \%$ frequency degradation under high temperature for all of our test cases. The recommended operating temperature of the FPGAs we use is within $-40^{\circ} \mathrm{C}$ to $85^{\circ} \mathrm{C}$. In our test cases, we use $100^{\circ} \mathrm{C}$ and $110^{\circ} \mathrm{C}$, which are above the upper limit of temperature, but not too high 


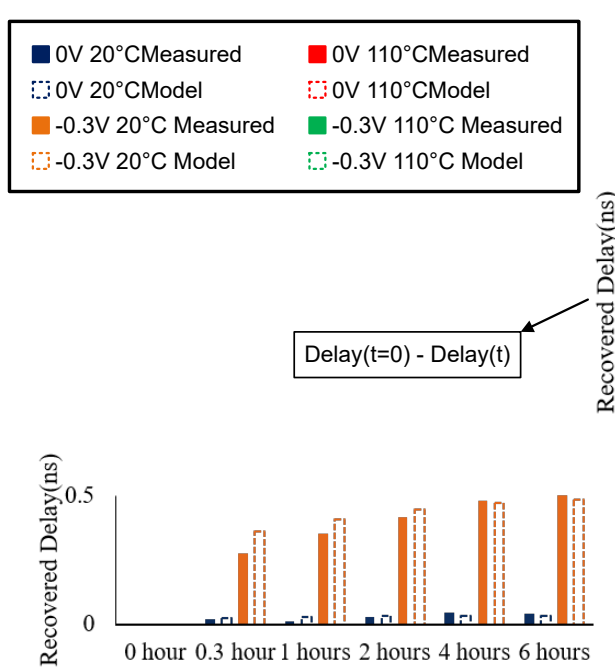

(a)

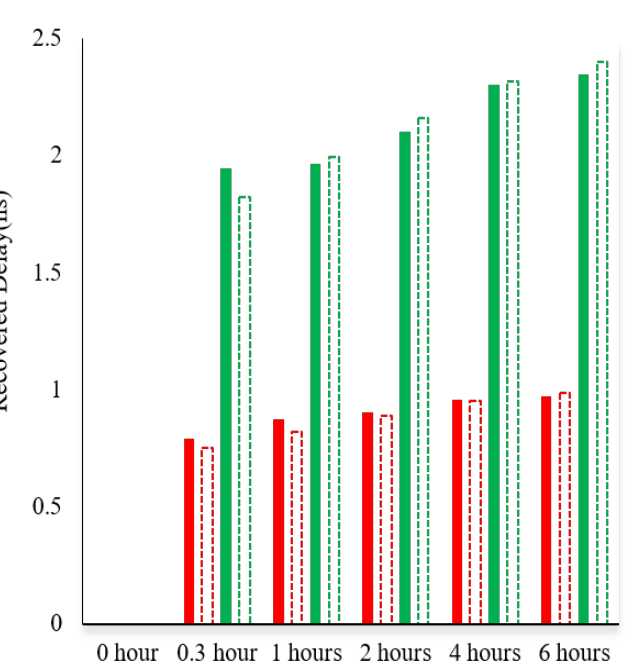

(b)

Fig. 4: Negative voltage-enabled active recovery after being stressed for 24 hours (Net delay increase is $\sim 3.24 n s$ ). $\mathrm{x}$-axis is the recovery time. (a) at $20^{\circ} \mathrm{C}$, (b) at $110^{\circ} \mathrm{C}$.

to prevent the chip from functioning. The FPGA chips are heated up or cooled down by a thermal chamber, which allows temperature fluctuation of $0.3^{\circ} \mathrm{C}$. Core voltage is provided by a DC power supply and its nominal value is $1.2 \mathrm{~V}$. A clock generator (on the interface board) provides the reference clock for the 16-b counter. Fig. 3b shows the measurement setup.

During the accelerated stress period, the CUT is kept under constant stress (DC stress) to "maximum" the wearout and it is enabled only every 20 minutes for checking the frequency. During recovery, a negative voltage and/or a high temperature of $110^{\circ} \mathrm{C}$ are applied. RO wakes up every 30 minutes for data sampling. The sampling time is less than 3s. All tests are carried out on a group of "fresh" FPGA chips that are within the same family. The chip that is stressed under regular operating conditions $\left(\mathrm{T}=20^{\circ} \mathrm{C}, V_{d d_{-} s t}=1.2 \mathrm{~V}\right)$ and/or recovered under passive recovery condition $\left(\mathrm{T}=20^{\circ} \mathrm{C}\right.$, $V_{d d_{-} r e c}=0 \mathrm{~V}$ ) is used as the baseline for comparisons.

\section{Test Results for Accelerated Self-Healing Techniques}

The test results for accelerated self-healing techniques are presented in this section. To make comparisons, we use recovered delay ( $\mathrm{RD}$ - reduced delay due to recovery) as the metric, and it can be calculated as $R D\left(t_{2}\right)=T_{d}\left(t_{1}\right)-T_{d}\left(t_{2}\right)=$ $\Delta T_{d}\left(t_{1}\right)-\Delta T_{d}\left(t_{2}\right)$, where $t_{2}>t_{1}$, and $\Delta T_{d}$ is the delay change.

1) Active Recovery by Negative Voltage: In modern chips, when certain blocks go to sleep, the supply voltage is usually gated to reduce leakage, and this also helps the recovery of wearout such as BTI, but this only results in passive recovery [43]. For the proposed active recovery (refer to Fig. 2 No. 2), we apply a negative voltage as supply voltage. The challenges for picking this negative voltage are: (a) Breakdown voltage limitation: the voltage must be at the level below the lateral pn-junction breakdown voltage; (b) Implementation feasibility: implementation of negative voltage will introduce area overhead; (c) Gate-induced Drain Leakage Current (GIDL) may introduce large leakage currents. In this test case, we picked a negative voltage of $-0.3 \mathrm{~V}$ that is validated to be still within the "safe" margin of the breakdown voltage and leakage. Fig. 4 compares the recovered delay over 6 hours $(1 / 4$ of the total stress time) when the temperature is set at $20^{\circ} \mathrm{C}$ and $110^{\circ} \mathrm{C}$, respectively. Model predictions based on Section III-A are also included in the figure. The coefficients used in the model are listed in Table I. Test results in Fig. 4 show that stressed chips rejuvenate faster with a negative supply voltage for both temperatures. By applying a negative voltage, the recovery rate is significantly increased even at room temperature.

2) Accelerated Recovery by High Temperature: High temperature not only accelerates wearout, this section will show that it will also accelerates recovery. Fig. 5 presents the recovered delay vs. temperature result under passive recovery $(0 \mathrm{~V})$ and active recovery $(-0.3 \mathrm{~V})$ conditions; in both cases, high temperature accelerates recovery. The proposed model in Section III-A accurately predicts this behavior, as also shown in the figure.

3) Summary: Table II summarizes the recovery percentage (Recovered delay/Net delay increase) for four test cases. It is worth to mention that in the accelerated \& active recovery case when both high temperature and negative voltage are applied to the system, about $72.4 \%$ of the degradation can be recovered within only $1 / 4$ of the stress time, and this also indicates that high temperature and negative voltage can "assist" each other during recovery and lead to the maximum recovered portion $(72.4 \%>>16.7 \%+28.7 \%)$. The recovered portion can be directly translated to the necessary design margin reduction. For example, with the accelerated $\&$ active recovery techniques, the design margin can be brought back to $27.6 \%$ of the original one within only a short period of recovery time.

\section{Frequency Dependence of Wearout and RECOVERY}

The proposed accelerated and active recovery techniques are able to rejuvenate the stressed chip significantly within a short time. While based on the physical trapping and de-trapping 


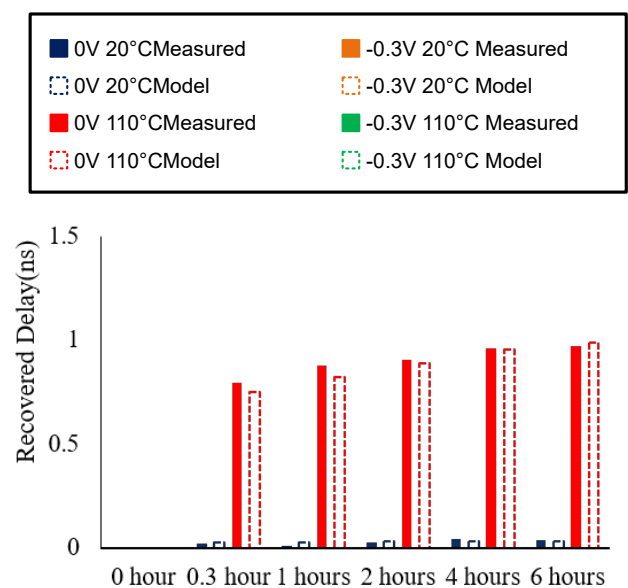

(a)

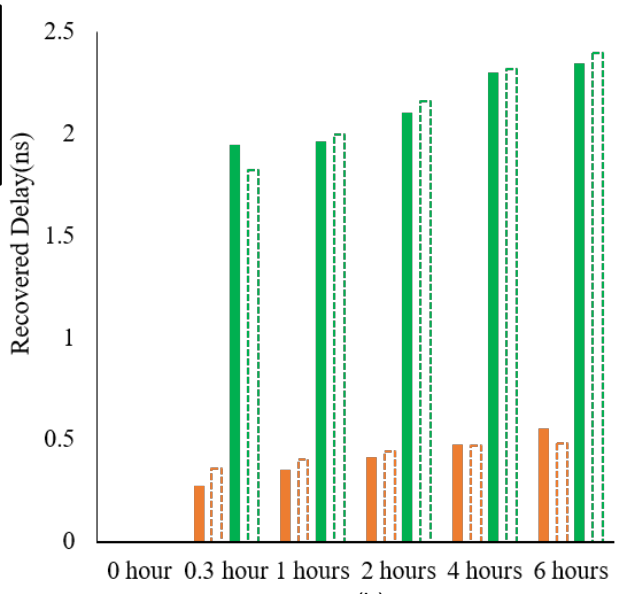

(b)

Fig. 5: High temperature-enabled accelerated recovery after being stressed for 24 hours (Net delay increase is $\sim 3.24 n s$ ). $\mathrm{x}$-axis is the recovery time. (a) under $0 \mathrm{~V}$ (passive recovery), (b) under $-0.3 \mathrm{~V}$ (active recovery).

TABLE II: Summary of the accelerated self-healing results for 6-hour recovery (recovery percentage \%)

\begin{tabular}{|c|c|c|c|}
\hline \hline Test Case & Sleep Condition & Measurement Results & Model Prediction \\
\hline Passive Recovery & $20^{\circ} \mathrm{C}$ and $0 \mathrm{~V}$ & $0.66 \%$ & $1 \%$ \\
\hline Active Recovery & $20^{\circ} \mathrm{C}$ and $-0.3 \mathrm{~V}$ & $16.7 \%$ & $14.4 \%$ \\
\hline Accelerated Recovery & $110^{\circ} \mathrm{C}$ and $0 \mathrm{~V}$ & $28.7 \%$ & $29.2 \%$ \\
\hline Accelerated \& Active Recovery & $110^{\circ} \mathrm{C}$ and $-0.3 \mathrm{~V}$ & $72.4 \%$ & $72.7 \%$ \\
\hline
\end{tabular}

nature of wearout discussed in Section II-A, and it is also validated by our experiments, there are still irreversible components (e.g.shown in Figure $1 \mathrm{~b}$ as $V_{\text {th }}$ net increase) that are hardly recovered within a reasonable time and will accumulate cycle by cycle, thus this will hurt the performance and increase the necessary guardband in a long term. In this section, we will look into this more in details by exploring the boundary between the reversible and irreversible parts of wearout and studying the frequency dependence of wearout and recovery by conducting tests on FPGAs. The same experimental setup in Section III-B will be used in this part ${ }^{2}$.

\section{A. Reversible vs. Irreversible Wearout}

1) Physics Perspective - Fast Traps vs. Slow Traps: Based on the trapping-detrapping theory [16], [18], the charge carriers need to gain sufficient kinetic energy to overcome a potential barrier necessary to break an interface state to be captured in the traps during trapping process. Here we define fast traps as those traps that have a high probability of trapping the charge carriers. These traps have a relatively lower trap energy barrier and are easier to be filled in a shorter time. On the contrary, slow traps are those have a higher trap energy barrier that is difficult for charge carriers to overcome. The principle of physics for de-trapping (recovery process) is that the trapped charge carriers (e.g. electrons for NMOS) have a certain probability to escape, with the probability being higher if their energy is higher and the trap energy barrier is lower, and vice-versa. Based on the statistical mechanics theory, the distribution of kinetic energies is proportional to the product

\footnotetext{
${ }^{2}$ Part of this work was originally published in [10]
}

of density of state and the Boltzmann distribution. The 3dimensional density of state is proportional to the energy, the energy distribution of electrons at room temperature $(300 \mathrm{~K})$ is plotted in Fig. 6a, which shows that majority of the electrons are at low energy in $\mathrm{meV}$ range, whereas the center energy of even the lowest energy of the trap is in order of several kT $(\sim 0.026 \mathrm{eV})$ [44]. This means that only a small fraction of electrons at the tail of the distribution could participate in the de-trapping process.

Illustrated in Fig. $6 \mathrm{~b}$ is the trapping and detrapping process for above two types of traps. Since fast traps have lower trap energy barrier, so it is easier for charge carriers to escape from them, and this leads to fast recovery, or reversible part of wearout. For the slow traps, the charge carriers need to overcome a higher trap energy barrier. As a result, it is very slow or even impossible for them to escape within a reasonable time, so these traps will cause the irreversible wearout. To give a first order estimation, if the trap energy is $100 \mathrm{meV}$ higher, the probability distribution goes down by a factor of $\exp (100 \mathrm{meV} / 20 \mathrm{emV}) \approx 50$. This indicates that the time it takes to de-trap goes up by about a factor of 50 for every $100 \mathrm{meV}$ increase in trap energy.

Temperature and voltage (electrical field) play an important role of determining energy of electrons. Fig. $6 c$ shows that by increasing the temperature, the energy distribution skews to the right, so the probability of de-trapping increases. Besides temperature, voltage could also affect the detrapping process through the electrical field. This indicates that the boundary between the reversible and irreversible wearout can even be affected by these factors.

2) Sequentiality of Reversible and Irreversible Wearout: As reversible wearout and irreversible wearout are mostly 


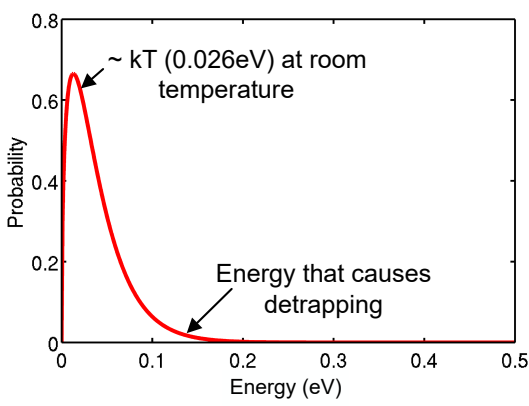

(a) Energy distribution of electrons at room temperature

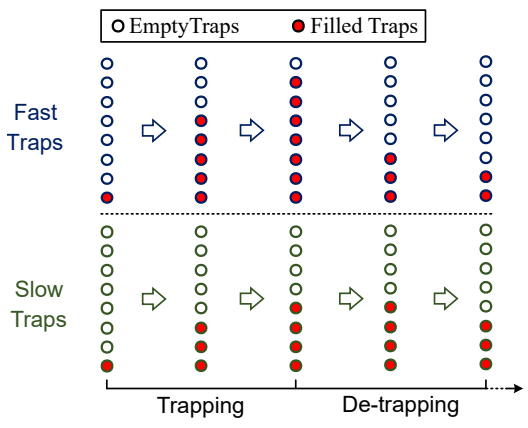

(b) Illustration of Fast traps vs. Slow traps

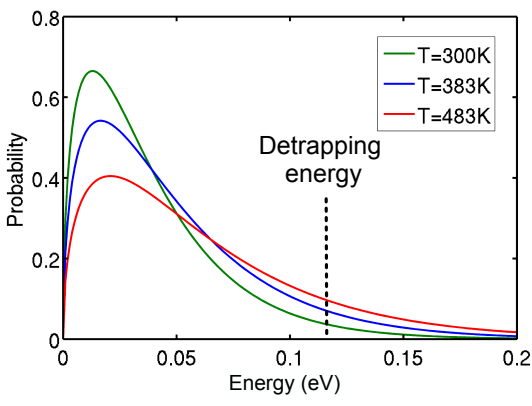

(c) Temperature affects the energy distribution and the boundary

Fig. 6: A physics perspective of irreversible wearout.

determined by fast traps and slow traps respectively. So there will be sequences when wearout happens due to the different trapping rate of the two. To further investigate this, a group of stress tests is conducted. Shown in Fig. 7a is the frequency change under two accelerated stress conditions with different stress voltages. It illustrates that both conditions follow similar wearout patterns. Firstly, reversible wearout kicks in, then the effect of reversible wearout levels off and irreversible wearout takes over - in time domain this is roughly seen as a steep slope followed by shallow slope during wearout.

Fig. $7 \mathrm{~b}$ shows a test case when a 6-hour accelerated \& active recovery $\left(110^{\circ} \mathrm{C},-0.3 \mathrm{~V}\right)$ process follows a 6-hour stress. The accelerated and active recovery techniques speed up the recovery process and even recover some parts that would otherwise be considered irreversible. In the time domain this can be roughly seen as a steep slope followed by a saturation (zero slope) once all the reversible part and part of irreversible part were recovered. Also, it is worth to mention that the Recovered Wearout is larger than the Reversible Wearout as shown in the figure, and this further demonstrates that the boundary between reversible and irreversible wearout is actually "soft" and can even be controllable.

3) Irreversible Wearout during Accelerated \& Active Recovery: As shown in Figure 8a, although that part of the irreversible wearout left by passive recovery could be recovered by the proposed accelerated and active recovery techniques, there are still irreversible components that will keep accumulating throughout the system lifetime. Figure $8 \mathrm{~b}$ is a test case for several cycles of stress and recovery. In each cycle, a 6hour accelerated stress is followed by a 6 -hour accelerated \& recovery (6 hours vs. 6 hours). IRn refers to the amount of irreversible wearout for the $n t h$ cycle. It shows in the figure that the recovery under accelerated conditions saturates in each cycle, and the irreversible wearout (IR) increases for the first few cycles and settles afterwards. A possible explanation for this behavior is that in the later cycles, some of the irreversible wearout from previous cycles starts to recover, and the accelerated recovery and stress can fully compensate each other. But it will not be fully recovered to the fresh state even with accelerated and active recovery techniques applied during each recovery interval.

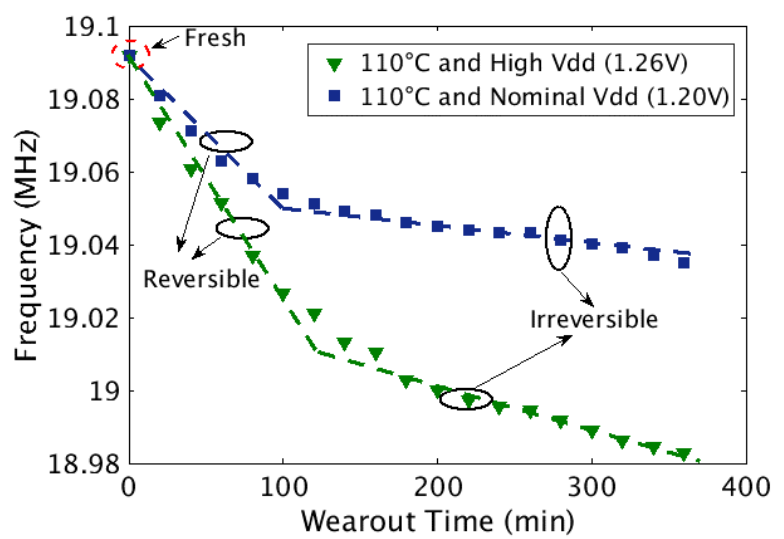

(a) Frequency degradation under two accelerated stress conditions

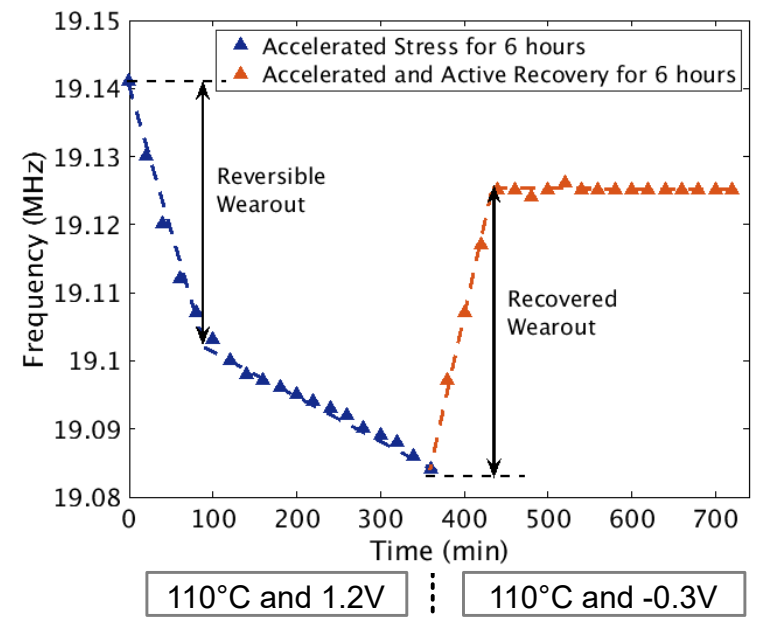

(b) Frequency change during wearout (6 hours) and accelerated \& active recovery (6 hours)

Fig. 7: Sequentiality of reversible and irreversible wearout.

\section{B. "Work hard, sleep well” for FULL Recovery}

1) Sleep with rejuvenation when getting tired: The whole process of wearout and (accelerated \& active) recovery can be compared to the biological world. For example, humans beings are active during daytime, with the body conducting activities and experiencing fatigue. During night time sleep, the body 


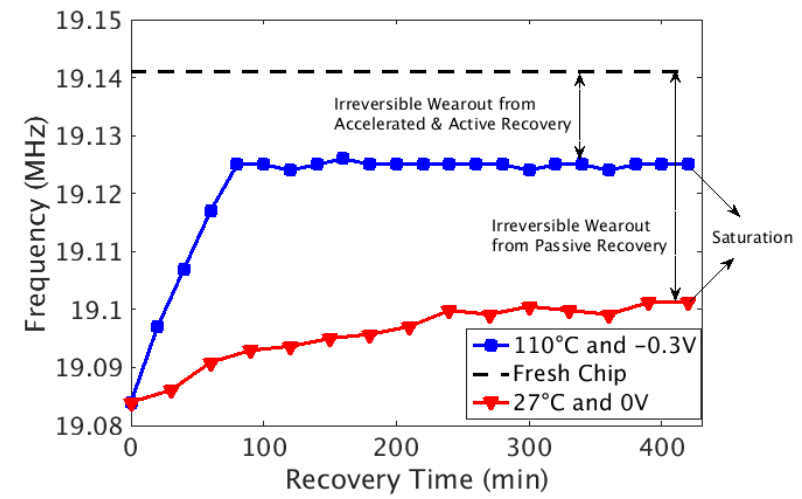

(a) Irreversible component under passive recovery and accelerated \& active recovery conditions

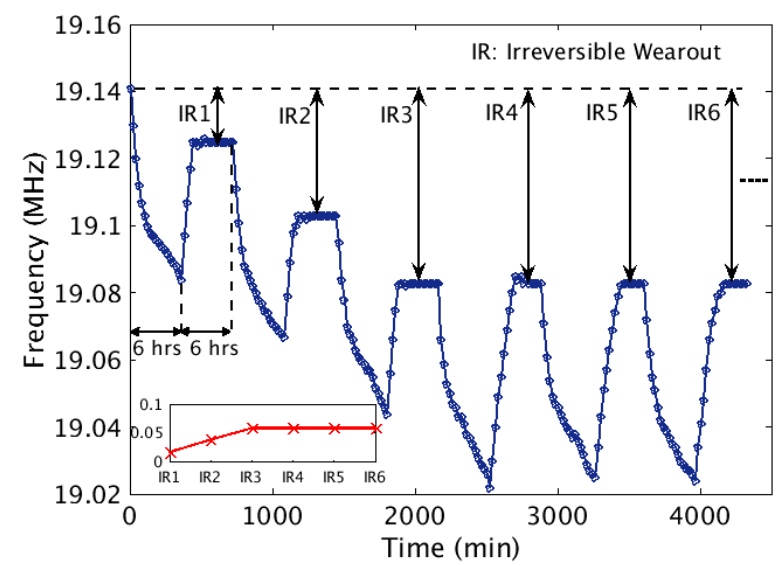

(b) Accumulation of the irreversible wearout cycle by cycle

Fig. 8: Irreversible wearout during accelerated \& active recovery.

goes through several active processes that are essential for the recovery of its full capabilities for the next day. If some organs experience heavy fatigue without in-time rest, part of the fatigue will be translated into some potential harms to the body, and will be hardly recovered. This is well known for athletes that need scheduled recovery periods after extensive workouts, with their athletic performance actually getting better after the rest periods. These biological fatigue and recovery schedules are not unlike those illustrated in Figure $8 \mathrm{~b}$. We thus borrow these ideas and apply to the electronic systems. The key idea is to stop the stress before irreversible effects get a chance to accumulate. The ideal strategy is thus to keep the circuit active only during the reversible phase of wearout until the irreversible wearout kicks in; thus the irreversible wearout becomes almost unobservable even in accelerated stress cases. To validate this idea, a set of tests with different "circadian rhythms" is conducted. All chips start from the fresh state. The total test time is 3 days for all test cases.

2) Measurement Results: Shown in Fig. 9 are measurement results. For all test cases except the $1 \mathrm{hr}$ vs. $1 \mathrm{hr}$ case, the accelerated active recovery has a period of saturation which indicates the irreversible parts of the wearout, and the irreversible parts accumulate in the first several cycles

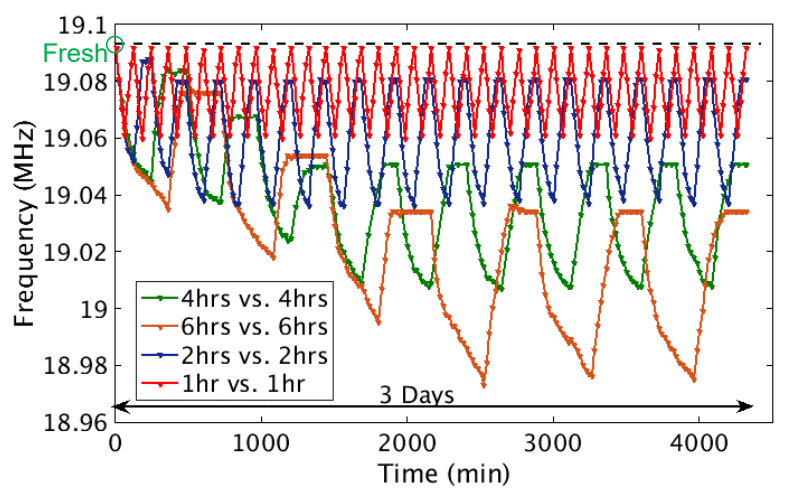

Fig. 9: Measurement results for four different "circadian rhythms".

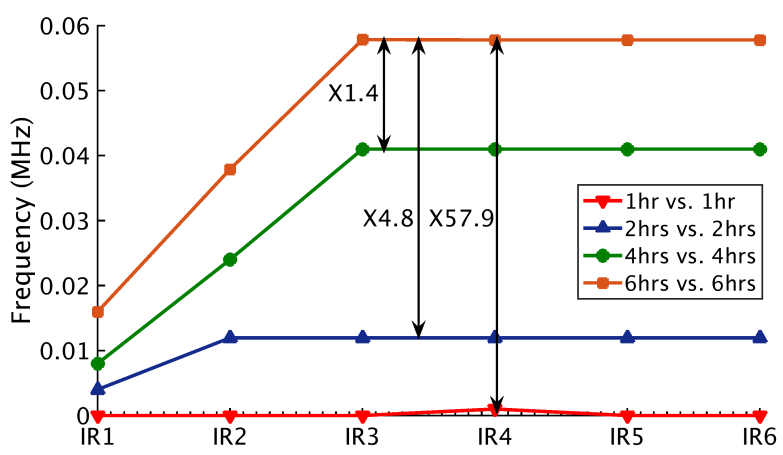

Fig. 10: Irreversible component accumulated during the first 6 cycles for four different scheduled stress and accelerated \& active recovery shifts (circadian rhythms).

and settle down in the following cycles. For the $1 \mathrm{hr} v \mathrm{vs} .1 \mathrm{hr}$ case, alternating phases of stress and accelerated recovery can completely compensate for each other, and after each accelerated and active recovery phase the chip can indeed start fresh. The irreversible part of wearout is totally avoided explicitly. Fig. 10 shows the accumulated irreversible wearout for the first 6 cycles under above four test conditions. It shows that the earlier the accelerated rejuvenation techniques are applied, the slower the irreversible wearout accumulate, which results in less permanent component. There is an optimal balance of stress and accelerate recovery (e.g. $1 \mathrm{hr}$ vs. $1 \mathrm{hr}$ in this accelerated stress case) which leads to almost no irreversible wearout.

Assume that the amount of frequency degradation under regular operating condition is the same as the accelerated case for 1 hour, the equivalent duration is about 31 hours under nominal voltage and room temperature based on the model proposed in Section III-A. As shown in Fig. 11, the identical optimal condition could be that the chip is active under regular operating conditions (room temperature and nominal $V d d$ ) for (at most) 31 hours, the accumulated wearout could be fully mitigated by a following 1 hour (or longer) accelerated and active recovery duration. 


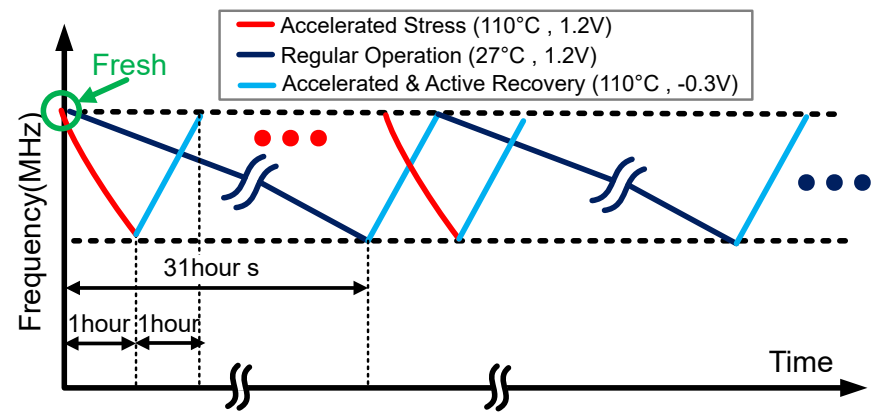

Fig. 11: An identical regular-operation use case (31hrs vs. 1hr) to the $1 \mathrm{hr}$ vs. $1 \mathrm{hr}$ accelerated stress case for FULL recovery

\section{Results \& AnAlysis}

\section{A. Necessary Design Margin \& Tracking Power Reduction}

The explored frequency dependent behaviors of wearout and (accelerated \& active) recovery provide great potentials of reducing the design margins significantly during the early design phase. As discussed in Section I, for the worst case design solution, to meet the timing requirement throughout the whole lifetime, guardbands need to be added (e.g. by oversizing transistors). Without the proposed periodic accelerated and active rejuvenation solutions, the margin needs to cover both reversible and irreversible wearout, and the irreversible part has to cover long time periods (typically years). Since the proposed strategy starts recovery before the irreversible wearout kicks in, the design margin only need to cover reversible wearout. Assume that the irreversible wearout at room temperature is the same as the one under the accelerated stress case (at $110^{\circ} \mathrm{C}$ ), and we define $\mathrm{AC}$ stress as the case when transistors switch between ON and OFF with a 50\% duty cycle, which gives the balanced stress and recovery during operation. Based on the model in Section III-A, the estimated design margin of 5 year and 10 year lifetime spans under DC and AC stress at room temperature is shown in Fig. 12. The proposed solution (1hr. vs. $1 \mathrm{hr}$ case) gives a necessary design margin reduction of at least $60 \mathrm{X}$ for all cases. In the $\mathrm{AC}$ stress case for a 10-year lifetime constraint, the design margin reduction is more than 100X. It also shows that as the lifetime constraint increases, the guardbands need to be relaxed (2X) correspondingly, while with the proposed strategy, the design margin stays almost the same (1X).

The alternative solutions for dealing with wearout are adaptive techniques where wearout sensors are used to track during the whole period of the lifetime. This means more tracking power. With the proposed strategy, the time for recovery is known ahead, wearout sensors only need to track during a short time (e.g. 31 hours as shown in Fig. 11) for the reversible part of wearout. Numerically the difference for the tracking power is between $O(\ln (31$ hours $))$ and $O(\ln (10$ years $))$ for a 10-year lifetime constraint, or about $\ln (2826) \sim 8 X$ reduction.

\section{B. Average Performance Improvement}

With the periodic accelerated and active rejuvenation, the system is guaranteed to run faster than the case when no

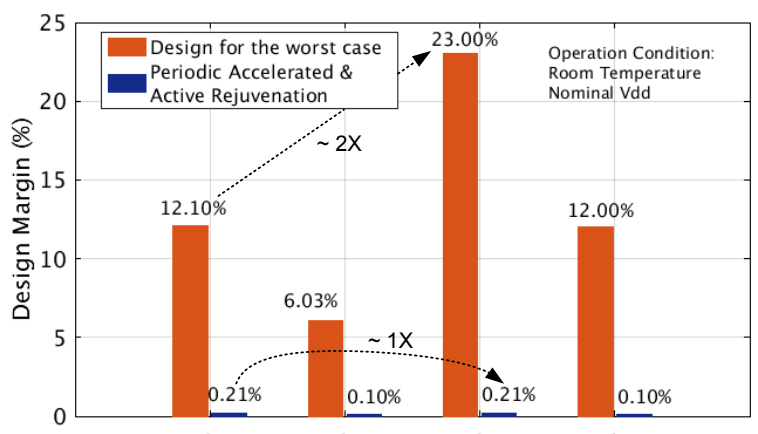

5 years $D C$ stress 5 years $A C$ stress 10 years $D C$ stress 10 years $A C$ stress

Fig. 12: Design margin estimation under different stress conditions.

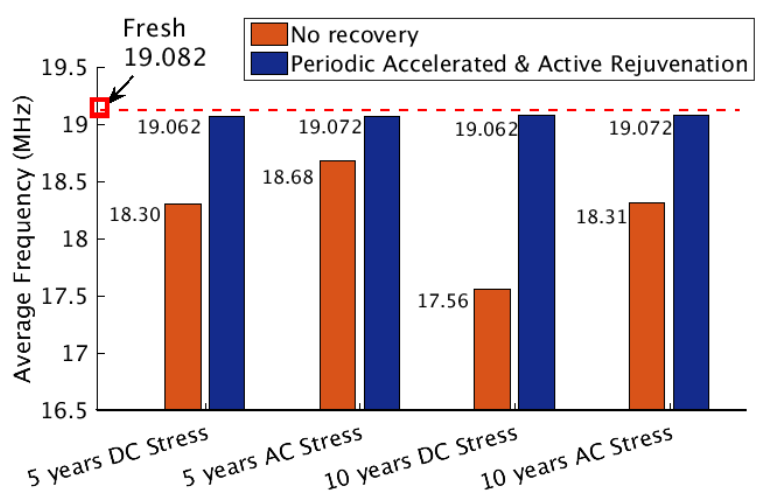

Fig. 13: Average performance under different stress conditions.

recovery is applied. We define that the average performance refers to the average of all performance values during operation time (wearout period). Figure 13 shows the predicted average performance improvement over the no-recovery solution under regular operation conditions (room temperature, nominal voltage) predicted by the model. As the lifetime constraint increases from 5 years to 10 years, the average performance for the proposed rejuvenation solution will keep almost the same, compared to the no-recovery case when the average performance scales down dramatically. In other words, the average performance improvement enabled by the proposed solution will increase significantly as the lifetime requirement increases. To give an example, for a 10-year lifetime span, the improvement can be as large as $\sim 9 \%$.

\section{High Temperature or Negative Voltage? or both?}

As shown in Section III and Section IV, high temperature of $110^{\circ} \mathrm{C}$ and negative voltage of $-0.3 \mathrm{~V}$ are picked and combined as effective accelerated self-healing to achieve significant metric improvements (recovery percentage, design margin reductions and average frequency improvement). While this combination shouldn't be fixed since different applications and physical locations on chip will have different available high temperature or negative voltage resources, so it is necessary to explore other possible combinations so that they will offer the flexibility for on chip implementations (will be discussed in the 


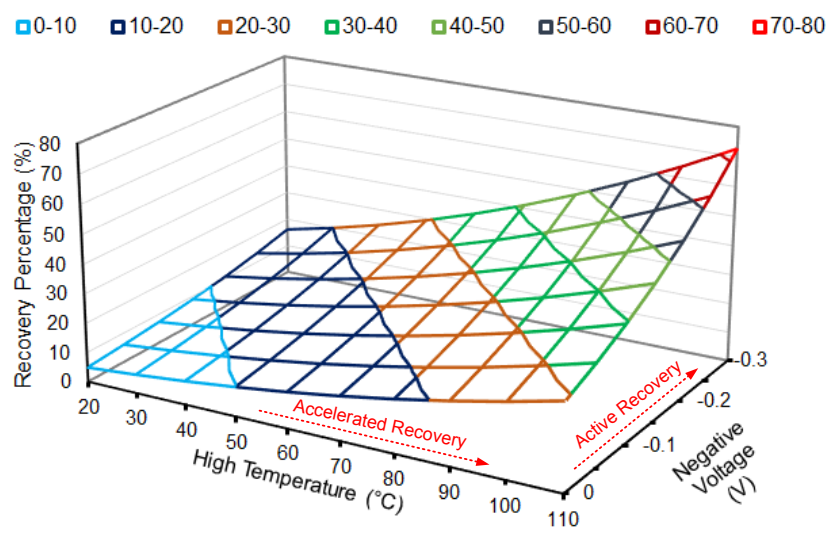

Fig. 14: Accelerated self-healing space exploration for a case when a 6-hour recovery follows a 24-hour accelerated stress.

following section). Fig. 14 shows the explored accelerated selfhealing space based on the proposed model in Section III-A. The same model parameters used in Section III-C are used here. We define "High Temperature" as the temperature above $20^{\circ} \mathrm{C}$ and "Negative Voltage" as any voltage values below $0 \mathrm{~V}$. Assume that the chip is stressed under high temperature of $110^{\circ} \mathrm{C}$ for 24 hours, the recovery percentage of $72.3 \%$ demonstrated in Section III is defined as the upper limit for the reversible wearout in this study. The surface plot presents the recovery percentage (recovered delay/net delay increase due to wearout) under different accelerated \& active recovery conditions for a 6-hour recovery period (long enough for reversible wearout to be fully recovered as shown in Fig. 7b). It turns out that the same recovery percentage can be achieved with multiple combinations of high temperature and negative voltage. For example, to achieve a recovery percentage of $\sim 30 \%$, the combinations could be $\left(50^{\circ} \mathrm{C},-0.3 \mathrm{~V}\right),\left(60^{\circ} \mathrm{C}\right.$, $0.25 \mathrm{~V}),\left(70^{\circ} \mathrm{C},-0.2 \mathrm{~V}\right),\left(80^{\circ} \mathrm{C},-0.15 \mathrm{~V}\right),\left(90^{\circ} \mathrm{C},-0.1 \mathrm{~V}\right),\left(100^{\circ} \mathrm{C}\right.$, $-0.05 \mathrm{~V})$ or $\left(110^{\circ} \mathrm{C}, 0 \mathrm{~V}\right)$. One observation is that the resulted recovery percentage by every $10^{\circ} \mathrm{C}$ of temperature increase is almost comparable to what is achieved by reduction of $0.05 \mathrm{~V}$ of the voltage. This offers the flexibility and possibilities of even "controlling" the recovery levels via voltages and temperatures. It also indicates the potentials of implementing the techniques under different part of the chips (with different available high temperatures and/or negative voltages) while achieving the same recovery percentage even though the onchip heat is not uniformly distributed or have fluctuations. Details of the implementations at different layers will be discussed in the following section.

\section{CLASH: CROSS-LAYER ACCELERATED SELF-HEALING IMPLEMENTATIONS}

The notion of cross-layer resilience enables design of reliable systems by implementing various techniques at different layers, and these techniques work together to achieve the optimal resilience levels with a low cost [2], [8]. Examples of such techniques could be cross-layer error prediction or detection for soft errors [4], run-time sensing and actuation [11] and so on. Orthogonal to what has existed, the proposed periodic accelerated self-healing techniques are able to "repair" the wearout completely and efficiently by fully taking advantage of the unique recovery and frequency dependence behaviors, and this will introduce another design dimension for optimizing the system resilience. This section presents a potential cross-layer accelerated self-healing (CLASH) system which integrates a set of such implementations from the circuit level to the system level.

\section{A. Circuit-level Implementations: On-chip Negative Voltage Generator and Heater}

1) On-chip Negative Voltage: To provide the on-chip negative voltage, a switch-capacitor charge-pump negative voltage generator that is based on [45] is designed and simulated in 28nm FD-SOI technology. Shown on the left half of Fig. 15 is the schematic of the generator. During the first half of the charge-pump cycle, clk1 is " 0 " and clk2 is "1", the flying capacitor $\mathrm{C} 1$ is charged to $V d d$. In the second half, clk1 is " 1 " and clk2 is " 0 ", this will lead to that the positive terminal of $\mathrm{C} 1$ is connected to ground and the negative terminal to Vout, $\mathrm{C} 1$ is in parallel with $\mathrm{C} 2$. The charge will be redistributed until it reaches the new balance. The value of Vout is mainly determined by the capacitance value of $\mathrm{C} 1$ and $\mathrm{C} 2(\mathrm{C} 1 / \mathrm{C} 2)$ and the clock frequency. Shown on the right half of the Fig. 15 is the simulated results. It shows that the generator outputs a stable voltage of $-300.6 \mathrm{mV}$ after a startup time of $638 \mathrm{~ns}$ under the clock frequency of $66.7 \mathrm{MHz}$. One of the most efficient ways of reducing ripples is to use non-overlapping clocking which is also implemented in this work and shown on the top left of figure. The resulted ripple is less than $1.45 \%$, which is good enough for the active recovery purpose. Fig. 16 presents one type of implementation which supports the negative voltage-enabled active recovery within a powergated block. An NMOS switch is added to the virtual power supply node for delivering the negative voltage when active recovery mode is activated (Signal Active Recovery = "1"). Since the capacitor $\mathrm{C} 2$ in the negative voltage generator (Fig. 15) acts as the decoupling capacitor (decap) explicitly, so no external decap for the negative voltage is needed. Thus the additional area overhead for a power gated block is only an NMOS switch. Since power gating is widely used in modern processors and embedded systems, so it is relatively easy to integrate the active recovery techniques into the existing infrastructures.

The estimated overall area of the negative voltage generator is less than $300 \mathrm{um}^{2}$. The power consumption is only about $64.47 \mathrm{uW}$, which can be further reduced by slowing down clock frequency and/or sizing the switches. Since there are only 4 transistors in the generator, the introduced leakage overhead is only $68.85 \mathrm{nW}$.

2) On-chip High Temperature: On-chip high temperature can be from two ways. The first one is by designing on-chip heating elements. There are work [46], [47] that proposed to use re-configurable blocks with high switching activities or small ring oscillators to generate heat for thermal-aware testing on FPGA. The results were promising and showed a very wide range of achievable temperatures by small heating 

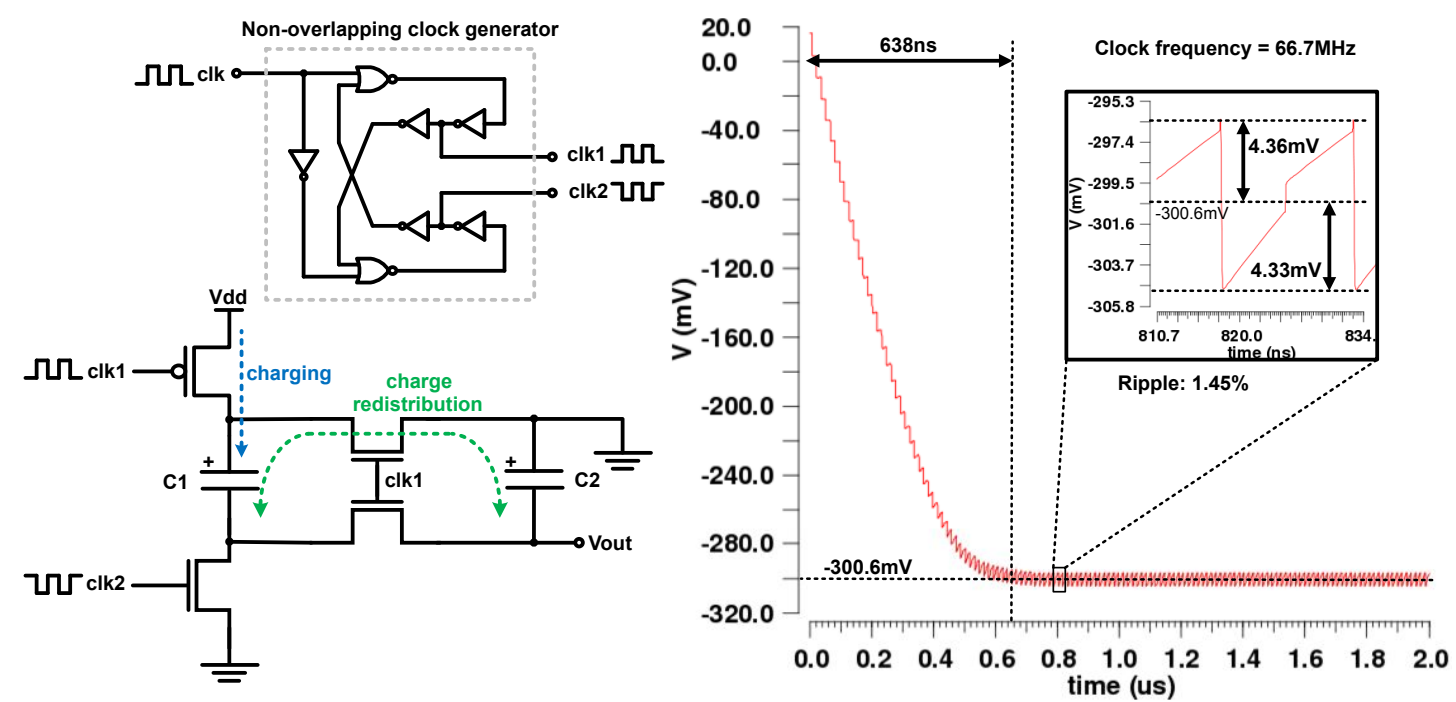

Fig. 15: Negative voltage generator and its simulated output $(\sim-0.3 V)$.

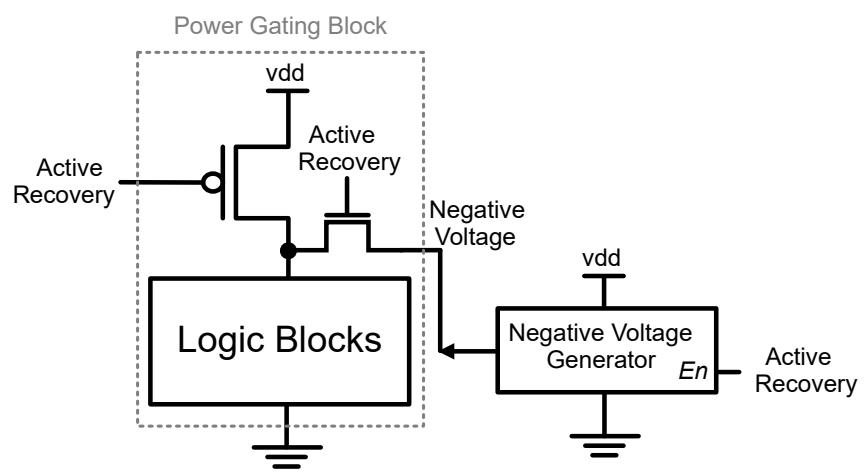

Fig. 16: A possible implementation with power gating.

blocks with a very high accuracy (variations are within $\pm 1^{\circ} \mathrm{C}$ ) [47]. So for our high temperature-enabled accelerated recovery purpose, similar blocks can be used as the heat generators that are enabled only during the recovery intervals. Fig. 17 presents two possible heater placement strategies. The first one (a) is ring-like, where core logic (such as the whole single core) can be surrounded by a "heater ring". The second solution (b) is to only place the heaters close to the critical path(s) which determines the overall performance of the systems. The first solution is relatively easy to implement, it needs more elements, also the center of the core might not be able to reach the desired healing temperature due to the physical distances. The second placement strategy is more effective since the heaters only need to heal the elements in critical path, and the amount of heaters is relatively less than the ring placement. The challenge is to have the right methodology to place them during the early design phase. One possible solution could be to embed the single element into a scan chain cell and place them during the DFT process.

Since each heat element includes only 2 gates as shown in Fig. 17, thus the introduced additional area and leakage are small (e.g. in $28 \mathrm{~nm}$ FDSOI technology, the X4 inverter takes only $0.39 \mathrm{um}^{2}$ of the area, and the leakage is only $410 \mathrm{pW}$ ). But

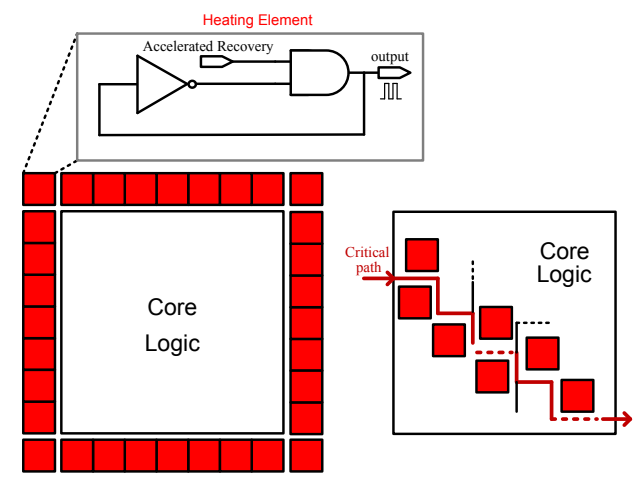

(a)

(b)

Fig. 17: Possible on-chip heater (based on the heating elements proposed in [47]) placement. (a) ring placement, (b) critical path placement.

it should be noted that the on-chip heating elements might not be suitable for all applications since the generated heat means power consumption, which will add to the energy budget. So careful design is necessary to balance the tradeoffs between energy during the sleep period and the improved metrics during run time. A more economical way of implementation is to utilize core level redundancy in a multicore system and have active cores act as "intrinsic on-chip heaters" for the neighboring asleep cores. This will be discussed as the second heating solution in the following section.

\section{B. Architecture-level Implementations: Dark Silicon and Core Redundancy}

In multicore or Network-on-Chip (NoC) systems which consist of hundreds or even thousands of identical cores, due to the TDP limitations, a significant amount of cores cannot be operated at the same time, and this leads to the so-called dark silicon problem [48]. Recent research [49] has pointed out that even with the latest FinFET technology and novel processor architectures, dark silicon issue still exists and stays 


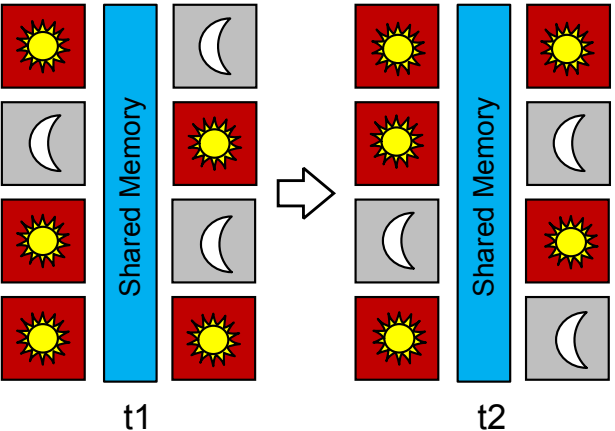

Fig. 18: A potential implementation in multi-core system.

as a big challenge. The dark silicon usually leads to some "redundant" core resources, and these resources can be a single core or a subset of the core. Existing works tried to leverage the redundancy by developing frameworks considering the amount of work and temperature variations and analyzing the the different redundancy arrangements [50], on top of these techniques, if these resources are scheduled and allocated in such a way that they can be healed by the generated heat from the active elements, the average lifetime of the whole system will be significantly improved. It has been shown that the temperature difference between the active regions and the inactive regions can be as large as $30^{\circ} \mathrm{C}$ in alpha processor and other modern many-core designs [51]. Thus the huge amount of generated heat by the active elements can be fully took advantages of. Fig. 18 illustrates a potential way of implementing in a simplified multicore system, where the sleeping cores (or resources) are always surrounded by the active cores (or resources), by switching the workload among different cores, these sleeping cores can be deeply rejuvenated.

This method is application dependent and also needs the support of the system scheduler. There will also be switching overhead, but this solution overcomes the potential energy overhead introduced from generating the on-chip heat and could be used together with the circuit level solutions to balance the tradeoffs. Details will be presented in Section VII.

\section{System-level Implementations: Right Balance of Wearout and Accelerated \& Active Rejuvenation}

As discussed in Section IV, the accelerated \& active recovery techniques might not be able to lead to full recovery without the proper scheduled wearout and recovery period due to existing of the irreversible component. Philosophically, there are two alternatives for scheduling recovery: reactive, when an integrated circuit (IC) or part of an IC has aged by a particular threshold amount [52], and proactive, in anticipation of future wearout. Reactive recovery is potentially more "economic" since it is only scheduled when needed. But it needs to track changing threshold voltages, and has the disadvantage of being unpredictable, thus potentially introducing performance and/or energy overheads at inopportune times, and likely leading to a smaller improvement in lifetime, and accumulates upfront more irreversible wearout thus leading to a lower expected performance and energy circuit operates more time in an

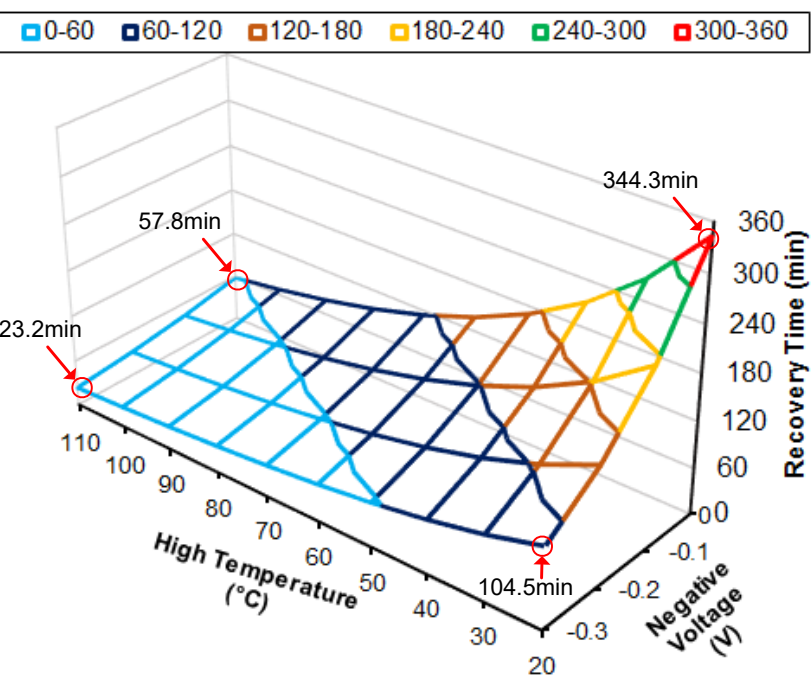

Fig. 19: Recovery time under different accelerated \& active recovery conditions after 12-hour constant stress under regular operation condition (room temperature, nominal Vdd).

wearout mode. While for proactive recovery, the recovery periods are scheduled ahead of any sign of wearout, is easier to implement. Thus the right balance of wearout and recovery means that the circuit or system could run (be active) under a higher level of performance as long as possible but could be rejuvenated back to the fresh state within a short sleep duration. It should be noted the 31-hour of regular operation vs. the 1-hour of accelerated \& active recovery demonstrated in Section IV-B2 should not be fixed, it differs depending on different technology, applications and use cases.

Based on experimental results and analysis in Section IV-B2 and Section $\mathrm{V}-\mathrm{C}$, wearout is a relatively slow process under regular operating conditions (without being accelerated), even the reversible part of the wearout usually takes at least one day under the constant stress (e.g. 31 hours), so the sleep period could be scheduled roughly in a daily (or several days) base the accelerated and active recovery follows a 1-day (or several days) period of being active. Here we define two use cases and discuss the possible scheduled patterns correspondingly.

- Use case 1: For applications like mobile devices, the scheduled active vs. sleep pattern could even follow human beings' circadian rhythms, for example, the devices are active during daytime for 12 hours, then during sleep, although certain blocks need to be still active for data retention, some critical performance-hungry components could be deeply rejuvenated for next day's "full speed". The length of the recovery time depends on the conditions (temperature and voltage). Assume that the system or is active for 12 hours under regular conditions (room temperature and nominal voltage). In the worst case, it is always under constant (DC) stress, but still in irreversible wearout region (within 31 hours). Based on the model in Section III-A and analysis in Section V-C, the required time for full recovery under different sleep condition is plotted in Fig. 19. It shows that recovery is very fast and needs about 23.2 minutes under the best condition 


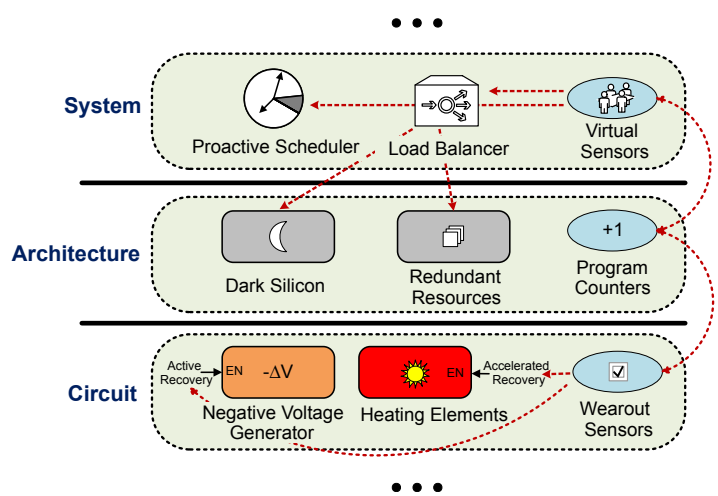

(a) Illustration of Cross-layer Accelerated Self-Healing

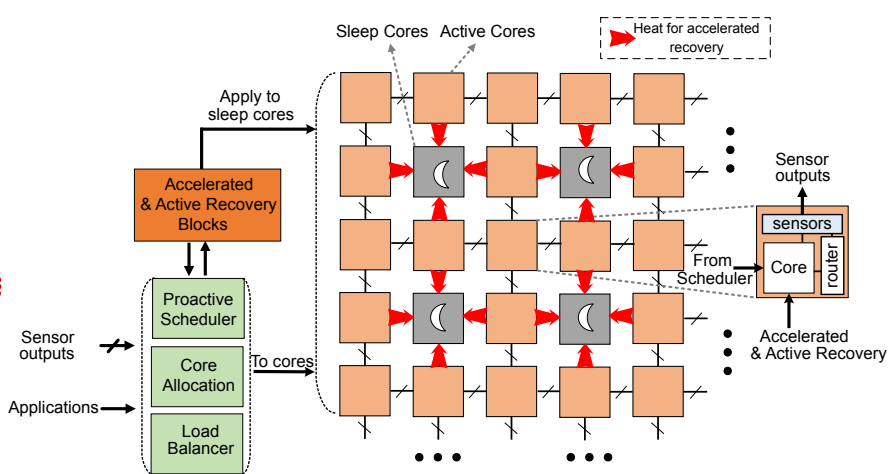

(b) A potential implementation of CLASH in a NoC system

Fig. 20: CLASH - Cross-Layer Accelerated Self-Healing System.

of $110^{\circ} \mathrm{C}$ and $-0.3 \mathrm{~V}$. For a slight higher temperature or negative voltage, the recovery can be much faster than the regular operating condition. For example, under $50^{\circ} \mathrm{C}$ and $-0.3 \mathrm{~V}$, it only needs about 57 minutes to fully recover to the fresh state. These devices could benefit from such short sleep intervals through periodic rejuvenation.

- Use case 2: For data center or server applications, although the system runs most of the time, and it might not be feasible to fully turn off the system as frequently as in mobile devices or personal PCs. While recent research [53]-[55] have proposed energy-efficient solutions that implement novel load balancing and/or scheduling algorithms so that the idle and lightly-loaded cores are able to be switched to sleep states, which can be utilized as the intrinsic sleep periods for the full recovery. During the sleep states, the core allocator and scheduler can work together to take advantages of the heat generated by the active nodes. The detailed analysis for the server and data center applications will be left as the future work.

\section{TRADEOFF ANALYSIS}

As discussed in the previous section, there will be overhead for each type of implementations, but these implementations at different system stack could be potentially integrated into a single system and work together to balance the tradeoffs among power, performance and resilience as shown in Fig. 20a. For example, generating heat only with on-chip heating elements or providing the negative voltages by the circuitlevel voltage generator together will cause additional power overhead during the sleep interval, and this will be expensive, especially for those energy constraint applications. Thus at the architecture level, the core allocator or load balancer can work together to assist the process by shifting the applications among different cores and making the right allocations (e.g. as shown in Fig. 18) so that the circuit level solution can "rest". While due to the existing of the cooling system, the generated heat might be released soon, thus the circuit level heating and negative voltage generator could restart again. At the upper level, the system scheduler leverages the tradeoffs and schedules the necessary accelerated self-healing periods proactively (e.g. following a pattern as discussed in Section VI-C). Key elements that enable the transparency between each layer are wearout sensors. Such sensors can be physical sensors (ring oscillator based [56] or meta-stable elements based [57]) at the circuit level, program counters at the architecture level, or virtual sensors at the system or application levels. The collaborative efforts from all layers will guarantee that the whole system will always run in a "refresh" mode and is almost "wearout-free" over the entire lifetime with a relatively low cost.

Illustrated in Fig. 20b is a potential implementation in a multicore system that consists of many identical cores, each of which includes several small wearout sensors (can be both circuit and architecture sensors shown in Fig. 20a) that monitor the core behaviors and send the wearout information back to the management units (scheduler, core allocator or load balancer) that determine how many cores and which core to disable based on the acceptable wearout levels, application behaviors and power/thermal constraints. These units will also enable the circuit and architecture level accelerated and active recovery blocks to apply the accelerated and recovery techniques to these sleep elements. Since most of the modern processors already include the wearout sensors and employ power gating techniques for power and thermal optimization purposes, there is a good opportunity to combine the proposed strategy with the existing real-time scheduling techniques and algorithms to achieve power, thermal and wearout optimization together. The strategy of employing shorter accelerated rejuvenation duration (hours) after a longer span (days) of reversible wearout can be introduced as a "negative turboboost" solution. With this method, the overall necessary design margins can be significantly reduced, and the system can perform for more of the time at higher levels of performance with optimal power efficiency.

\section{CONCLUSION}

In this paper, we demonstrate that wearout can be reversed significantly through a set of accelerated self-healing techniques. By studying the recovery behaviors of wearout extensively based on hardware measurements, we explored the frequency dependent behaviors which show that certain stress/recovery schedules can (almost) completely eliminate 
the irreversible wearout, thus allowing significant reductions in necessary design margins and average performance improvements. To enable the on-chip implementations of the accelerated self-healing and fully utilize the frequency dependent behaviors, we propose several low-cost implementations across the digital system hierarchy, which shows the big potentials of introducing the accelerated self-healing as a key design knob to achieve the optimal resilience effectively. As the future work, we will further study the tradeoffs among power, performance and resilience with the introduction of the accelerated self-healing at different layers. Several implementation challenges at the architecture level and system level will also be addressed in the future.

\section{ACKNOWLEDGMENT}

This work was supported in part by NSF Grant No. CCF1255907, SRC task 2410.001 and the Center for Future Architectures Research (C-FAR), one of six SRC STARnet Centers, sponsored by MARCO and DARPA. The authors would also like to thank Prof. Wayne Burleson (University of Massachusetts Amherst) and Alec Roelke (University of Virginia) for helpful discussions and valuable feedback.

\section{REFERENCES}

[1] K. Bernstein, D. J. Frank, A. E. Gattiker, W. Haensch, B. L. Ji, S. R. Nassif, E. J. Nowak, D. J. Pearson, and N. J. Rohrer, "High-performance cmos variability in the $65-\mathrm{nm}$ regime and beyond," IBM journal of research and development, vol. 50, no. 4.5, pp. 433-449, 2006.

[2] J. Henkel, L. Bauer, N. Dutt, P. Gupta, S. Nassif, M. Shafique, M. Tahoori, and N. Wehn, "Reliable on-chip systems in the nano-era: Lessons learnt and future trends," in Proceedings of the 50th Annual Design Automation Conference. ACM, 2013, p. 99.

[3] H. Hong, J. Lim, H. Lim, and S. Kang, "Lifetime reliability enhancement of microprocessors: Mitigating the impact of negative bias temperature instability," ACM Computing Surveys (CSUR), vol. 48, no. 1, p. 9, 2015.

[4] S. Mitra, K. Brelsford, and P. N. Sanda, "Cross-layer resilience challenges: Metrics and optimization," in Design, Automation \& Test in Europe Conference \& Exhibition (DATE), 2010. IEEE, 2010, pp. 1029_ 1034.

[5] W. Wang, S. Yang, S. Bhardwaj, R. Vattikonda, S. Vrudhula, F. Liu, and Y. Cao, "The impact of nbti on the performance of combinational and sequential circuits," in Proceedings of the 44th annual Design Automation Conference. ACM, 2007, pp. 364-369.

[6] R. Wang, P. Ren, C. Liu, S. Guo, and R. Huang, "Understanding nbtiinduced dynamic variability in the nano-reliability era: From devices to circuits," in Physical and Failure Analysis of Integrated Circuits (IPFA), 2015 IEEE 22nd International Symposium on the. IEEE, 2015, pp. $119-121$.

[7] N. P. Carter, H. Naeimi, and D. S. Gardner, "Design techniques for cross-layer resilience," in Proceedings of the Conference on Design, Automation and Test in Europe. European Design and Automation Association, 2010, pp. 1023-1028.

[8] S. Mitra, P. Bose, E. Cheng, C.-Y. Cher, H. Cho, R. Joshi, Y. M. Kim, C. R. Lefurgy, Y. Li, K. P. Rodbell et al., "The resilience wall: Crosslayer solution strategies," in VLSI Technology, Systems and Application (VLSI-TSA), Proceedings of Technical Program-2014 International Symposium on. IEEE, 2014, pp. 1-11.

[9] K. Kang, S. Gangwal, S. P. Park, and K. Roy, "Nbti induced performance degradation in logic and memory circuits: how effectively can we approach a reliability solution?" in Proceedings of the 2008 Asia and South Pacific Design Automation Conference. IEEE Computer Society Press, 2008, pp. 726-731.

[10] X. Guo and M. R. Stan, "Work hard, sleep well - avoid irreversible ic wearout with proactive rejuvenation," in 201621 st Asia and South Pacific Design Automation Conference (ASP-DAC), Jan 2016, pp. 649654.
[11] S. Sarma, N. Dutt, N. Venkatasubramanian, A. Nicolau, and P. Gupta, "Cyberphysical system-on-chip (cpsoc): Sensoractuator rich self-aware computational platform," University of California Irvine, Tech. Rep. CECS TR-13-06, 2013.

[12] S. V. Kumar, C. H. Kim, and S. S. Sapatnekar, "Adaptive techniques for overcoming performance degradation due to aging in digital circuits," in Proceedings of the 2009 Asia and South Pacific Design Automation Conference. IEEE Press, 2009, pp. 284-289.

[13] H. Mostafa, M. Anis, and M. Elmasry, "Nbti and process variations compensation circuits using adaptive body bias," Semiconductor Manufacturing, IEEE Transactions on, vol. 25, no. 3, pp. 460-467, 2012.

[14] L. Zhang and R. P. Dick, "Scheduled voltage scaling for increasing lifetime in the presence of nbti," in Design Automation Conference, 2009. ASP-DAC 2009. Asia and South Pacific. IEEE, 2009, pp. 492497.

[15] S. Gupta and S. S. Sapatnekar, "Employing circadian rhythms to enhance power and reliability," ACM Transactions on Design Automation of Electronic Systems (TODAES), vol. 18, no. 3, p. 38, 2013.

[16] Y. Cao, J. Velamala, K. Sutaria, M. S.-W. Chen, J. Ahlbin, I. Sanchez Esqueda, M. Bajura, and M. Fritze, "Cross-layer modeling and simulation of circuit reliability," Computer-Aided Design of Integrated Circuits and Systems, IEEE Transactions on, vol. 33, no. 1, pp. 8-23, 2014.

[17] T. Grasser, T. Aichinger, G. Pobegen, H. Reisinger, P.-J. Wagner, J. Franco, M. Nelhiebel, and B. Kaczer, "The 'permanent' component of nbti: composition and annealing," in Reliability Physics Symposium (IRPS), 2011 IEEE International. IEEE, 2011, pp. 6A-2.

[18] S. Mahapatra, Fundamentals of Bias Temperature Instability in MOS Transistors: Characterization Methods, Process and Materials Impact, DC and AC Modeling. Springer, 2015, vol. 52.

[19] S. Zafar, Y. Kim, V. Narayanan, C. Cabral Jr, V. Paruchuri, B. Doris, J. Stathis, A. Callegari, and M. Chudzik, "A comparative study of nbti and pbti (charge trapping) in sio2/hfo2 stacks with fusi, tin, re gates," in VLSI Technology, 2006. Digest of Technical Papers. 2006 Symposium on. IEEE, 2006, pp. 23-25.

[20] V. Huard, M. Denais, and C. Parthasarathy, "Nbti degradation: From physical mechanisms to modelling," Microelectronics Reliability, vol. 46 , no. 1 , pp. $1-23,2006$.

[21] J. B. Velamala, K. Sutaria, T. Sato, and Y. Cao, "Physics matters: statistical aging prediction under trapping/detrapping," in Proceedings of the 49th Annual Design Automation Conference. ACM, 2012, pp. 139-144.

[22] S. Gupta and S. S. Sapatnekar, "Gnomo: Greater-than-nominal v dd operation for bti mitigation," in Design Automation Conference (ASPDAC), 2012 17th Asia and South Pacific. IEEE, 2012, pp. 271-276.

[23] J. Abella, X. Vera, and A. Gonzalez, "Penelope: The nbti-aware processor," in Microarchitecture, 2007. MICRO 2007. 40th Annual IEEE/ACM International Symposium on. IEEE, 2007, pp. 85-96.

[24] X. Chen, Y. Wang, Y. Cao, Y. Ma, and H. Yang, "Variation-aware supply voltage assignment for simultaneous power and aging optimization," Very Large Scale Integration (VLSI) Systems, IEEE Transactions on, vol. 20, no. 11, pp. 2143-2147, 2012.

[25] A. Tiwari and J. Torrellas, "Facelift: Hiding and slowing down aging in multicores," in Microarchitecture, 2008. MICRO-41. 2008 41st IEEE/ACM International Symposium on. IEEE, 2008, pp. 129-140.

[26] N. Shah, R. Samanta, M. Zhang, J. Hu, and D. Walker, "Built-in proactive tuning system for circuit aging resilience," in Defect and Fault Tolerance of VLSI Systems, 2008. DFTVS'08. IEEE International Symposium on. IEEE, 2008, pp. 96-104.

[27] T. Siddiqua and S. Gurumurthi, "Nbti-aware dynamic instruction scheduling," in Proceedings of the 5th Workshop on Silicon Errors in Logic-System Effects. Citeseer, 2009.

[28] L. Li, Y. Zhang, J. Yang, and J. Zhao, "Proactive nbti mitigation for busy functional units in out-of-order microprocessors," in Proceedings of the Conference on Design, Automation and Test in Europe. European Design and Automation Association, 2010, pp. 411-416.

[29] D. M. Ancajas, K. Chakraborty, and S. Roy, "Proactive aging management in heterogeneous nocs through a criticality-driven routing approach," in Proceedings of the Conference on Design, Automation and Test in Europe. EDA Consortium, 2013, pp. 1032-1037.

[30] H. Reisinger, O. Blank, W. Heinrigs, W. Gustin, and C. Schlünder, "A comparison of very fast to very slow components in degradation and recovery due to nbti and bulk hole trapping to existing physical models," Device and Materials Reliability, IEEE Transactions on, vol. 7, no. 1, pp. 119-129, 2007.

[31] J. Shin, V. Zyuban, P. Bose, and T. M. Pinkston, "A proactive wearout recovery approach for exploiting microarchitectural redundancy to ex- 
tend cache sram lifetime," in ACM SIGARCH Computer Architecture News, vol. 36, no. 3. IEEE Computer Society, 2008, pp. 353-362.

[32] T. Siddiqua and S. Gurumurthi, "Recovery boosting: A technique to enhance nbti recovery in sram arrays," in VLSI (ISVLSI), 2010 IEEE Computer Society Annual Symposium on. IEEE, 2010, pp. 393-398.

[33] A. Bansal and J.-J. Kim, "Power napping technique for accelerated negative bias temperature instability (nbti) and/or positive bias temperature instability (pbti) recovery," Jul. 21 2015, uS Patent 9086865.

[34] T. Aichinger, M. Nelhiebel, and T. Grasser, "On the temperature dependence of nbti recovery," Microelectronics Reliability, vol. 48, no. 8, pp. $1178-1184,2008$.

[35] A. A. Katsetos, "Negative bias temperature instability (nbti) recovery with bake," Microelectronics Reliability, vol. 48, no. 10, pp. 1655-1659, 2008.

[36] G. Pobegen, T. Aichinger, M. Nelhiebel, and T. Grasser, "Understanding temperature acceleration for nbti," in Proc. Intl. Electron Devices Meeting (IEDM), 2011, pp. 27-3.

[37] B. Djezzar, H. Tahi, A. Benabdelmoumene, A. Chenouf, M. Goudjil, and Y. Kribes, "On the permanent component profiling of the negative bias temperature instability in p-mosfet devices," Solid-State Electronics, vol. 106, pp. 54-62, 2015.

[38] X. Guo, W. Burleson, and M. Stan, "Modeling and experimental demonstration of accelerated self-healing techniques," in Proceedings of the 51st Annual Design Automation Conference. ACM, 2014, pp. $1-6$.

[39] K. Ramakrishnan, S. Suresh, N. Vijaykrishnan, M. J. Irwin, and V. Degalahal, "Impact of nbti on fpgas," in VLSI Design, 2007. Held jointly with 6th International Conference on Embedded Systems., 20th International Conference on. IEEE, 2007, pp. 717-722.

[40] G. Kömürcü, A. E. Pusane, and G. Dündar, "Effects of aging and compensation mechanisms in ordering based ro-pufs," Integration, the VLSI Journal, vol. 52, pp. 71-76, 2016.

[41] M. Naouss and F. Marc, "Design and implementation of a low cost test bench to assess the reliability of fpga," Microelectronics Reliability, vol. 55, no. 9, pp. 1341-1345, 2015.

[42] S. Velusamy, W. Huang, J. Lach, M. Stan, and K. Skadron, "Monitoring temperature in fpga based socs," in Computer Design: VLSI in Computers and Processors, 2005. ICCD 2005. Proceedings. 2005 IEEE International Conference on. IEEE, 2005, pp. 634-637.

[43] A. Calimera, A. Macii, E. Macii, and M. Poncino, "Power-gating for leakage control and beyond," in Circuit Design for Reliability. Springer, 2015, pp. 175-205.

[44] V. Huard, F. Cacho, X. Federspiel, and P. Mora, "Hot-carrier injection degradation in advanced cmos nodes: a bottom-up approach to circuit and system reliability," in Hot Carrier Degradation in Semiconductor Devices. Springer, 2015, pp. 401-444.

[45] Maxim, "Max 1044/icl7660 datasheet." [Online]. Available: https: //www.maximintegrated.com/en/datasheet/index.mvp/id/1017

[46] P. Weber, M. Zagrabski, P. Musz, K. Kepa, M. Nikodem, and B. Wojciechowski, "Configurable heat generators for fpgas," in Thermal Investigations of ICs and Systems (THERMINIC), 2014 20th International Workshop on. IEEE, 2014, pp. 1-4.

[47] A. Amouri, J. Hepp, and M. Tahoori, "Built-in self-heating thermal testing of fpgas," IEEE Transactions on Computer-Aided Design of Integrated Circuits and Systems, vol. PP, no. 99, pp. 1-1, 2016.

[48] H. Esmaeilzadeh, E. Blem, R. S. Amant, K. Sankaralingam, and D. Burger, "Dark silicon and the end of multicore scaling," in Computer Architecture (ISCA), 2011 38th Annual International Symposium on. IEEE, 2011, pp. 365-376.

[49] J. Henkel, H. Khdr, S. Pagani, and M. Shafique, "New trends in dark silicon," in Design Automation Conference (DAC), 2015 52nd ACM/EDAC/IEEE. IEEE, 2015, pp. 1-6.

[50] L. Huang and Q. Xu, "Characterizing the lifetime reliability of manycore processors with core-level redundancy," in Computer-Aided Design (ICCAD), 2010 IEEE/ACM International Conference on. IEEE, 2010, pp. 680-685.

[51] C. Zhuo, K. Chopra, D. Sylvester, and D. Blaauw, "Process variation and temperature-aware full chip oxide breakdown reliability analysis," Computer-Aided Design of Integrated Circuits and Systems, IEEE Transactions on, vol. 30, no. 9, pp. 1321-1334, 2011.

[52] R. A. Ashraf, A. Al-Zahrani, N. Khoshavi, R. Zand, S. Salehi, A. Roohi, M. Lin, and R. F. DeMara, "Reactive rejuvenation of cmos logic paths using self-activating voltage domains," in Circuits and Systems (ISCAS), 2015 IEEE International Symposium on. IEEE, 2015, pp. 2944-2947.

[53] A. Gandhi, M. Harchol-Balter, and M. A. Kozuch, "Are sleep states effective in data centers?" in Green Computing Conference (IGCC), 2012 International. IEEE, 2012, pp. 1-10.
[54] A. Paya and D. Marinescu, "Energy-aware load balancing and application scaling for the cloud ecosystem," IEEE Transactions on Cloud Computing, vol. PP, no. 99, pp. 1-1, 2015.

[55] P. Bogdan, S. Garg, and U. Y. Ogras, "Energy-efficient computing from systems-on-chip to micro-server and data centers," in Green Computing Conference and Sustainable Computing Conference (IGSC), 2015 Sixth International. IEEE, 2015, pp. 1-6.

[56] T.-H. Kim, R. Persaud, and C. H. Kim, "Silicon odometer: An onchip reliability monitor for measuring frequency degradation of digital circuits," IEEE Journal of Solid-State Circuits, vol. 43, no. 4, pp. 874880,2008

[57] X. Guo and M. Stan, "Mcpens: Multiple-critical-path embeddable nbti sensors for dynamic wearout management," in Proceedings of the 11th Workshop on Silicon Errors in Logic-System Effects. Citeseer, 2015.

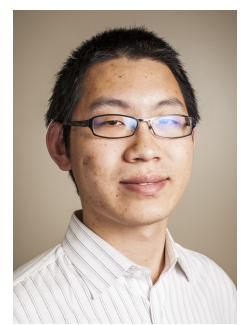

Xinfei Guo received the B.S. degree in Microelectronics from Xidian University, Xi'an, China, in 2010 and the M.S. degree in Electrical and Computer Engineering from the University of Florida, Gainesville, FL, in 2012. Currently, he is a Ph.D. candidate in Computer Engineering at the University of Virginia, Charlottesville, VA.

$\mathrm{He}$ has a broad interest in digital circuit and microarchitectures. His current research focus on Reliability (Wearout and Accelerated Recovery Techniques), Cross-layer power and reliability co-design methodology, Low-power and Energy-efficient design. He is a student member of the IEEE and ACM, and received the A. Richard Newton Young Student Fellowship and the Achievement Award in 2013 and 2010

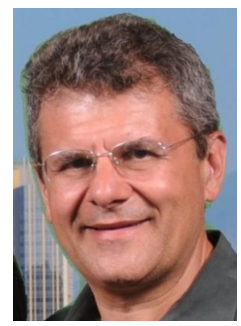

Mircea R. Stan received the Ph.D. (1996) and the M.S. (1994) degrees in Electrical and Computer Engineering from the University of Massachusetts at Amherst and the Diploma (1984) in Electronics and Communications from "Politehnica" University in Bucharest, Romania. Since 1996 he has been with the Charles L. Brown Department of Electrical and Computer Engineering at the University of Virginia, where he is now a professor. Prof. Stan is teaching and doing research in the areas of high-performance low-power VLSI, temperature-aware circuits and architecture, embedded systems, spintronics, and nanoelectronics. He leads the High-Performance Low-Power (HPLP) lab and is a co-director of the Center for Automata Processing (CAP). He has more than eight years of industrial experience, has been a visiting faculty at UC Berkeley in 2004-2005, at IBM in 2000, and at Intel in 2002 and 1999.

He has received the NSF CAREER award in 1997 and was a co-author on best paper awards at ISQED 2008, GLSVLSI 2006, ISCA 2003 and SHAMAN 2002. He was the chair of the VLSI Systems and Applications Technical Committee (VSA-TC) of IEEE CAS in 2005-2007, general chair for ISLPED 2006 and for GLSVLSI 2004, technical program chair for NanoNets 2007 and ISLPED 2005, and on technical committees for numerous conferences. He is a Senior Editor for the IEEE Transactions on Nanotechnology since 2014, and was an AE for the IEEE Transactions on Nanotechnology in 2012-2014, IEEE Transactions on Circuits and Systems Systems I in 20042008 and for the IEEE Transactions on VLSI Systems in 2001-2003. He was Guest Editor for the IEEE Computer special issue on Power-Aware Computing in December 2003 and a Distinguished Lecturer for the IEEE Circuits and Systems (CAS) Society in 2012-2013 and 2004-2005, and for the Solid-State Circuits Society (SSCS) in 2007-2008. Prof. Stan is a Fellow of the IEEE, a member of ACM, and of Eta Kappa Nu, Phi Kappa Phi and Sigma Xi. 